\title{
Costly Interpretation of Asset Prices
}

\author{
Jordi Mondria, Xavier Vives and Liyan Yang*
}

January 2020

\begin{abstract}
We propose a model in which investors cannot costlessly process information from asset prices. At the trading stage, investors are boundedly rational and their interpretation of prices injects noise into the price, generating a source of endogenous noise trading. Our setup predicts price momentum and yields excessive return volatility and excessive trading volume. In an overall equilibrium, investors optimally choose sophistication levels by balancing the benefit of beating the market against the cost of acquiring sophistication. There can exist strategic complementarity in sophistication acquisition, leading to multiple equilibria.
\end{abstract}

Keywords: Investor sophistication, price momentum, asset prices, complementarity

*Mondria: Department of Economics, University of Toronto, 150 St. George Street, Toronto, Ontario, M5S3G7, Canada; E-mail: jordi.mondria@utoronto.ca. Vives: IESE Business School, Avinguda Pearson, 21 08034 Barcelona, Spain.Yang: Rotman School of Management, University of Toronto, 105 St. George Street, Toronto, M5S3E6, ON, Canada; E-mail: liyan.yang@rotman.utoronto.ca. We thank George-Marios Angeletos, Efstathios Avdis, Jess Benhabib, Snehal Banerjee, Larry Blume, Patrick Bolton, Bradyn Breon-Drish, Matthijs Breugem, Georgy Chabakauri, Pierre Chaigneau, Amil Dasgupta, Shaun William Davies, Evan Dudley, Bernard Dumas, David Easley, Thierry Foucault, Simone Galperti, Diego García, Itay Goldstein, Naveen Gondhi, David Hirshleifer, Zhiguo He, Yu Hou, Zhen Huo, Philippe Jehiel, Alexandre Kohlhas, Jennifer La'o, John Leahy, Mina Lee, Samuel Lee, Xuewen Liu, Igor Makarov, Thomas Mertens, Jianjun Miao, Jordi Mondria, Christian Opp, Emre Ozdenoren, Alessandro Pavan, Ryan Riordan, Oleg Rytchkov, Francesco Sangiorgi, Jan Schneemeier, Karl Shell, Vasiliki Skreta, Günter Strobl, Edward D. Van Wesep, Laura Veldkamp, Venky Venkateswaran, Pengfei Wang, Yajun Wang, Pierre-Olivier Weill, Yizhou Xiao, Wei Xiong, Ming Yang, Kathy Yuan, Jing Zeng, Yao Zeng, and participants at various seminars and conferences for helpful comments. Mondria and Yang gratefully acknowledge the financial support from the Social Sciences and Humanities Research Council of Canada. Vives acknowledges the financial support of the Ministry of Science, Innovation and Universities with project PGC2018-096325-B-I00 (MCIU/AEI/FEDER, UE). 


\section{Introduction}

Data can be viewed as information only after it has been analyzed. Interpreting data is often costly in terms of effort and other resources, which is particular true for market data given the complexity of modern financial markets. In the existing frameworks - such as the noisy rational expectations equilibrium (REE) model (e.g., Grossman and Stiglitz, 1980; Hellwig, 1980; Vives, 2008) and the recent REE-disagreement hybrid models (e.g., Banerjee, 2011) - investors perfectly comprehend the price function and thus can costlessly read into the asset price to uncover valuerelevant information. Apparently, such an argument requires a high degree of sophistication on the part of market participants. ${ }^{1}$ What if interpreting price information is costly and investors commit errors in the inference process? How to determine the sophistication levels of investors in interpreting asset prices? How does investor sophistication affect market prices and trading volume? In this paper, we propose a behavioral model to address these questions.

In our model, a continuum of investors interact with each other in two periods $(t=0$ and 1$)$. At date 1, investors trade on private information in a financial market. As in the standard REE, the asset price aggregates information and investors make inference from the price. However, at the trading stage, investors are boundedly rational and do not fully understand how to read information from prices. A fully sophisticated investor would extract the best signal possible from the price (which is endogenously determined in equilibrium), while a less sophisticated investor introduces noise in interpreting the price. After investors form their beliefs based on the personalized price signals, they behave as Bayesian given their own beliefs and optimize accordingly. Through market clearing, investors' optimal asset demands in turn endogenously determine the equilibrium price function and hence the best price signal (i.e., the "truth" in investors' personalized price signals).

At date 0, investors optimally choose their sophistication levels to maximize ex ante expected

\footnotetext{
${ }^{1}$ As discussed by Guesnerie (1992), this comprehension is broadly justified in two ways: the "eductive" justification that relies on the understanding of the logic of the situation faced by economic agents and that is associated with mental activity of agents aiming at "forecasting the forecasts of others;" and the "evolutive" justification that emphasizes the learning possibilities offered by the repetition of the situation and that is associated with the convergence of several versions of learning processes. See Section 7.1 in Vives (2008).
} 
utilities understanding that at date 1 they will be boundedly rational in reading information from prices. On the one hand, increasing sophistication reduces the bounded rationality at the later trading stage, which therefore benefits investors ex ante. On the other hand, acquiring sophistication is costly. For instance, if we think of investors as individual investors, then in order to become more sophisticated, investors may need better education/training (which will cost wealth) or simply need to think harder (which will be involved with mental costs). The optimal sophistication level is determined by balancing the benefit from reduced bounded rationality against the cost of sophistication acquisition.

We first analyze the equilibrium in the date-1 financial market, which can be viewed as an REE extended with bounded rationality. We find that costly price interpretation can inject noise into the price system. Specifically, in our setting, the equilibrium price is a linear function of the asset fundamental and a noise term. The fundamental term comes from aggregating investors' private value-relevant information, which is the root reason why investors care to learn from the price. The noise term in the price arises from a common error in investors' personalized price signals, which is meant to capture the idea that in processing price data, investors may suffer a common cognitive error (such as "sentiment" / "misperception"). Compared to the standard REE, costly interpretation of prices leads to price momentum (future returns depend positively on the current price), excessive return volatility, and excessive trading volume, which is consistent with the existing empirical evidence (e.g., Jegadeesh and Titman (1993) and Moskowitz, Ooi and Pedersen (2012) on momentum; Shiller (1981) and LeRoy and Porter (1981) on excess volatility; and Odean (1999) and Barber and Odean (2000) on excessive trading).

After analyzing the date-1 financial market equilibrium, we return to date 0 and examine how sophistication levels are determined in an overall equilibrium. The incentive to acquire sophistication comes primarily from beating the average sophistication level across the market, which allows the investor to interpret the price better and trade better (i.e., more likely to buy low and sell high). We analyze two types of sophistication choices by investors: (1) sophistication levels to tame the exposure to the common sentiment and (2) sophistication levels to curb idiosyncratic errors of processing prices. We find that sentiment sophistication choice can exhibit 
strategic complementarity, leading to the possibility of multiple equilibria.

We then extend our setting to allow exogenous noise trading. In this extension, returns can exhibit either momentum or reversal, depending on the size of the exogenous noise trading. This is due to the interaction between two forces. First, exogenous noise trading tends to cause returns to exhibit reversal: large noise demand pushes price too high and thus, a high price predicts a future price decline (see Banerjee et al., 2009 and Ch. 4 in Vives, 2008). Second, costly interpretation of prices leads to underreaction of prices to information, giving rise to price momentum. The second effect dominates if and only if the size of noise trading is small. Nonetheless, independent of the size of noise trading, acquiring sophistication about taming sentiment can always lead to strategic complementarity and multiple equilibria.

We finally compare our model to four alternative existing theories that are conceptually related to our story: supply information (Ganguli and Yang, 2009), dismissive traders (Banerjee, 2011), cursed traders (Eyster, Rabin and Vayanos, 2019), and operation risk (Basak and Buffa, 2019). We show that supply information and operation risk deliver reversal, while dismissiveness and cursedness can deliver price momentum. Relative to dismissiveness and cursedness, our theory predicts different patterns on other variables such as trading volume (see next section for details).

\section{Related Literature}

A recent literature explores environment complexity that makes agents fail to account for the informational content of other players' actions in game settings. Eyster and Rabin (2005) develop the concept of "cursed equilibrium," which assumes that each player correctly predicts the distribution of other players' actions, but underestimates the degree to which these actions are

correlated with other players' information. Esponda and Pouzo (2016) propose the concept of "Berk-Nash equilibrium" to capture that people can have a possibly misspecified view of their environment. Although these models are cast in a game theoretical framework, the spirit of our financial market model is similar. In our model, investors' interactions are mediated by an asset 
price, which can be viewed a summary statistic for all the other players' actions.

Eyster, Rabin and Vayanos (2019) have applied the cursed equilibrium concept to a financial market setting. In their setting, an investor is a combination of a fully rational REE investor (who correctly reads information from the price) and a naive Walrasian investor (who totally neglects the information in the asset price). Thus, the notion of "cursedness" in Eyster et al. (2019) is conceptually related to the notion of "sophistication/attention" in our setting, since both notions aim to capture the fact that investors sometimes partially ignore information contained in asset prices. ${ }^{2}$ Our paper complements Eyster et al. (2019) in three important ways. First, their central results refer to explaining trading volume. For example, they show that as the number of traders diverges to infinity, the total trading volume goes to infinity in their framework. Instead, at the trading stage, we conduct a comparative static analysis with respect to investors' sophistication level, rather than with respect to the number of traders. This exercise allows us to compare our setting to a fully REE benchmark. Moreover, this exercise helps to differentiate our framework from Eyster et al. (2019) in terms of testable volume predictions. Eyster et al. (2019) predict that trading volume always increases with cursedness (i.e., decreases with sophistication). By contrast, volume is either increasing or hump-shaped in sophistication in our setting. Second and more importantly, our setting has an extra stage to determine the equilibrium level of investor sophistication, which generates novel theory results such as strategic complementarity in sophistication acquisition. ${ }^{3}$ In contrast, Eyster et al. (2019) do not explore these issues. Third, our analysis incorporates a common error in interpreting prices, which generates a form of endogenous noise trading. This result leads to the complementarity result in the sophistication acquisition stage, and again, these features are absent in Eyster et al. (2019).

Banerjee, Kaniel and Kremer (2009) and Banerjee (2011) have combined REE and disagreement frameworks to allow investors to underestimate the precision of other investors' private in-

\footnotetext{
${ }^{2}$ See Section 5 of Eyster et al. (2019) for extensive evidence that people do not sufficiently heed the information content of others' behavior and of financial markets. Addoum and Murfin (2017) recently document that equity market participants fail to account for information reflected in publicly posted loan prices.

${ }^{3}$ Recently, Angeletos and Sastry (2019) find a related result of strategic complementarity and multiplicity with rational inattention: When other agents pay less attention, prices are more confusing, and one agent finds it harder to pay attention in a setting of complexity.
} 
formation (and hence labeled as "dismissiveness" models). A dismissive investor can be roughly viewed as a combination of a fully sophisticated and a naive agent, and thus conceptually related to our investors at the trading stage. As we show in Section 7, in a dismissiveness model, returns exhibit momentum only when investors' risk aversion is sufficiently small. In addition, dismissiveness and our model deliver different volume predictions: dismissive volume decreases with sophistication, while in our setting, volume is either increasing or hump-shaped in sophistication. Basak and Buffa (2019) have considered models of operation risk, where investors add idiosyncratic noise into their optimal demand. In Section 7, we explore a setting of operation risk extended with common noise and show that this type of models deliver return reversal.

Banerjee, Davis and Gondhi (2019) have recently proposed a framework to allow investors to choose their beliefs and study when some empirically relevant behavioral biases such as overconfidence and dismissiveness naturally arise in equilibrium. To the extent that behavioral biases are negatively related to sophistication, this model is conceptually related to ours. Nonetheless, the trade-offs and so mechanisms in the two models are different and complementary. In our setting, investors would like to be fully sophisticated but infeasible because of the exogenous cost. In contrast, in Banerjee et al. (2019), investors often optimally choose not to be fully sophisticated, because wrong beliefs benefit investors by offering anticipated utility.

\section{A Model of Costly Interpretation of Asset Prices}

\subsection{Setup}

We consider an economy with three dates, $t=0,1$ and 2 . At $t=1$, two assets are traded in a competitive market: a risk-free asset and a risky asset. The risk-free asset has a constant value of 1 and is in unlimited supply. The risky asset is traded at an endogenous price $\tilde{p}$ and is in zero supply. It pays an uncertain cash flow $\tilde{v}$ at date 2 , where $\tilde{v} \sim N\left(0, \tau_{v}^{-1}\right)$ with $\tau_{v}>0$.

There is a continuum $[0,1]$ of investors who derive expected utility from their date- 2 wealth. They have constant-absolute-risk-aversion (CARA) utility with a risk aversion coefficient of $\gamma$. 
Investors have fundamental information and trade on it. Specifically, prior to trading, investor $i$ is endowed with the following private signal about the risky asset payoff $\tilde{v}$ :

$$
\tilde{s}_{i}=\tilde{v}+\tilde{\varepsilon}_{i}, \text { with } \tilde{\varepsilon}_{i} \sim N\left(0, \tau_{\varepsilon}^{-1}\right),
$$

where $\tau_{\varepsilon}>0$. We will refer to $\tilde{v}$ as "fundamentals."

Each investor has two selves, self 0 and self 1 , who make decisions at dates 0 and 1 respectively. Self 0 does not observe any of the realizations in the later date- 1 financial market, but at date 0 builds an understanding of how the market works. Self 0 instructs self 1 to extract fundamental information from the price using the best signal $\tilde{s}_{p}=c_{0}+c_{1} \tilde{p}$, where $c_{0}$ and $c_{1}$ are endogenous constants (that are implied by the equilibrium price price function). Self 1 , however, is boundedly rational and adds receiver noise to the intercept $c_{0}$ of the price signal $\tilde{s}_{p}{ }^{4}$ Specifically, self 1 adds noise to $\tilde{s}_{p}$ and observes

$$
\tilde{s}_{p, i}=\left(c_{0}+\frac{1}{\sqrt{\tau_{u, i}}} \tilde{u}+\frac{1}{\sqrt{\tau_{e, i}}} \tilde{e}_{i}\right)+c_{1} \tilde{p}
$$

where $\tilde{u} \sim N(0,1)$ is a sentiment error common for all investors, ${ }^{5} \tilde{e}_{i} \sim N(0,1)$ is an individual processing error and $\left(\tilde{v},\left\{\tilde{\varepsilon}_{i}\right\}, \tilde{u},\left\{\tilde{e}_{i}\right\}\right)$ are mutually independent. Self 0 at date 0 chooses the loadings $\frac{1}{\sqrt{\tau_{u}, i}}$ and $\frac{1}{\sqrt{\tau_{e}, i}}$ to sentiment $\tilde{u}$ and to individual processing error $\tilde{e}_{i}$ taking into account a cost function $C\left(\tau_{u, i}, \tau_{e, i}\right)$. When these loadings are small, self 1 adds little noise in the price interpretation and investors are sophisticated. Therefore, these loadings (inversely) measure investors' sophistication levels in handling sentiment error and handling individual processing error. Self 1 at date 1 chooses the investment $D_{i}$ in the risky asset to maximize her subjective

\footnotetext{
${ }^{4}$ Noise is only added to the intercept $c_{0}$ because it is the simplest possible additive noise. Hence, we assume that self 1 observes the correct slope $c_{1}$.

${ }^{5}$ For instance, in describing the speculative trading activities in Chinese stock market, a recent Financial Times article states: "Speculative decisions to buy or sell, on the other hand, are driven by market technicals, investment fads...speculative investing tends to be driven by expected changes in the market consensus, rather than by expected shifts in economic growth prospects." ("Fundamentals simply do not matter in China's stock markets," Financial Times, January 14, 2020, https://www.ft.com/content/2362a9a0-3479-11ea-a6d3-9a26f8c3cba4) These "investment fads" or "market consensus" can be broadly interpreted as the sentiment term $\tilde{u}$ in our setting.
} 
expected utility of ex post wealth

$$
\tilde{W}_{i}=(\tilde{v}-\tilde{p}) D_{i}-C\left(\tau_{u, i}, \tau_{e, i}\right)
$$

where we normalize her initial wealth level to 0 and take $\tau_{u, i}$ and $\tau_{e, i}$ as given. Self 1 is a price taker who infers information from the price $\tilde{p}$, although adding noise in the inference process. ${ }^{6}$

We assume that self 0 cannot force self 1 to use a specific demand function. This intends to capture the idea that self 1 is responsible for trading and adds processing errors to the instructions on how to extract information from the price passed by self 0 . If self 0 is able to instruct self 1 to implement demand schedules, this treatment shares the spirit similar to "operation risk" studied by Basak and Buffa (2019). In Section 7.4, we compare our model with operation risk and show that these two models deliver different predictions (e.g., our model predicts price momentum, while operation risk predicts return reversal).

\subsection{Timeline and Equilibrium Concept}

The timeline of our economy is as follows:

$t=0$ : Self 0 of each investor chooses $\tau_{u, i}$ and $\tau_{e, i}$ to maximize ex ante utility.

$t=1$ : Self 1 is boundedly rational in reading information from the price. Self 1 of each investor receives the private fundamental signal $\tilde{s}_{i}$ and the price signal $\tilde{s}_{p, i}$, and submits demand schedules. This implies that the demand schedule is $D\left(\tilde{p} ; \tilde{s}_{i}, \tilde{s}_{p, i}\right)$. Market clears at price $\tilde{p}$.

$t=2$ : Asset payoff $\tilde{v}$ is realized, and investors get paid and consume.

The overall equilibrium in our model is composed of a date-1 trading equilibrium in the financial market and a date-0 sophistication determination equilibrium. In the date-1 financial market equilibrium, self 1 of each investor maximizes her conditional subjective expected utility

\footnotetext{
${ }^{6}$ Although throughout the entire paper we take a behavioral interpretation based on individual investors, we can alternatively interpret the two selves of our investors as the research department (self 0) and trading desk (self 1) of an investment institution. The trading desk is responsible for trading assets and it relies on the institution's research department to have an understanding on how to generate information from prices. Research departments describe the procedure on how to extract the best signal from prices in the form of research reports, but trading desks add noise in comprehending the procedures in the reports.
} 
and the asset market clears for given sophistication levels $\tau_{u, i}$ and $\tau_{e, i}$. This equilibrium determines the price function and hence the best price signal $\tilde{s}_{p}$. In the sophistication determination stage, self 0 of each investor optimally chooses the sophistication levels $\tau_{u, i}$ and $\tau_{e, i}$ to maximize her ex-ante expected utility taking into account future equilibrium demands. In Section 4, we will first consider a financial market equilibrium taking investors' sophistication levels $\left\{\tau_{u, i}\right\}_{i \in[0,1]}$ and $\left\{\tau_{e, i}\right\}_{i \in[0,1]}$ as given. In Section 5, we will then deal with the overall equilibrium and the determination of sophistication levels.

\section{Financial Market Equilibrium}

At date 1, taking as given the sophistication levels $\tau_{u, i}$ and $\tau_{e, i}$, self 1 of each investor $i$ chooses investment $D_{i}$, that is, a demand function $D\left(\tilde{p} ; \tilde{s}_{i}, \tilde{s}_{p, i}\right)$ to maximize

$$
E\left[-e^{-\gamma(\tilde{v}-\tilde{p}) D_{i}} \mid \tilde{s}_{i}, \tilde{s}_{p, i}\right]
$$

The CARA-normal setting implies that investor $i$ 's demand for the risky asset is

$$
D\left(\tilde{p} ; \tilde{s}_{i}, \tilde{s}_{p, i}\right)=\frac{E\left(v \mid \tilde{s}_{i}, \tilde{s}_{p, i}\right)-\tilde{p}}{\gamma \operatorname{Var}\left(\tilde{v} \mid \tilde{s}_{i}, \tilde{s}_{p, i}\right)},
$$

where $E\left(\tilde{v} \mid \tilde{s}_{i}, \tilde{s}_{p, i}\right)$ and $\operatorname{Var}\left(\tilde{v} \mid \tilde{s}_{i}, \tilde{s}_{p, i}\right)$ are the conditional expectation and variance of $\tilde{v}$ given information $\left\{\tilde{s}_{i}, \tilde{s}_{p, i}\right\}$. In (5), we have explicitly incorporated $\tilde{s}_{p, i}$ in the demand function to reflect the informational role of the price (i.e., the price helps to predict $\tilde{v}$ ) and used $\tilde{p}$ to capture the substitution role of the price (i.e., a higher price directly leads to a lower demand). Here, the dependence of $D\left(\tilde{p} ; \tilde{s}_{i}, \tilde{s}_{p, i}\right)$ on the price $\tilde{p}$ in (5) reflects the fact that the investor knows that purchasing one unit of the asset costs $\tilde{p}$, while her learning on fundamentals operates through the private signal $\tilde{s}_{p, i}$ or "price interpretation."

The financial market clears as follows:

$$
\int_{0}^{1} D\left(\tilde{p} ; \tilde{s}_{i}, \tilde{s}_{p, i}\right) d i=0 \text { almost surely. }
$$


This market-clearing condition, together with demand function (5), will determine an equilibrium price function:

$$
\tilde{p}=p(\tilde{v}, \tilde{u})
$$

where $\tilde{v}$ and $\tilde{u}$ come from the aggregation of signals $\tilde{s}_{i}$ and $\tilde{s}_{p, i}$. In equilibrium, price function (7) will endogenously determine the informational content in the best signal $\tilde{s}_{p}$.

A financial market equilibrium for given sophistication levels $\left\{\tau_{u, i}\right\}_{i \in[0,1]}$ and $\left\{\tau_{e, i}\right\}_{i \in[0,1]}$ is characterized by a price function $p(\tilde{v}, \tilde{u})$ and demand function $D\left(\tilde{p} ; \tilde{s}_{i}, \tilde{s}_{p, i}\right)$, such that:

1. $D\left(\tilde{p} ; \tilde{s}_{i}, \tilde{s}_{p, i}\right)$ is given by $(5)$, which maximizes investors' conditional subjective expected utilities given their date-1 beliefs;

2. The market clears almost surely, i.e., equation (6) holds;

3. Investors' date-1 beliefs are given by (1) and (2), where $\tilde{s}_{p}$ in (2) is implied by the equilibrium price function $p(\tilde{v}, \tilde{u})$.

\subsection{Equilibrium Construction}

We consider a linear price function as follows:

$$
\tilde{p}=a_{v} \tilde{v}+a_{u} \tilde{u}
$$

where the coefficients $a_{v}$ and $a_{u}$ are endogenous. By equation (8), provided that $a_{v} \neq 0$ (which is true in equilibrium), the best price signal that a fully sophisticated investor can achieve is

$$
\tilde{s}_{p}=\frac{\tilde{p}}{a_{v}}=\tilde{v}+\alpha \tilde{u} \text { with } \alpha \equiv \frac{a_{u}}{a_{v}} .
$$

Note that the first equation in (9) indicates that the best price signal $\tilde{s}_{p}$ is linear in $\tilde{p}$; that is, $\tilde{s}_{p}=c_{0}+c_{1} \tilde{p}$, with coefficients $c_{0}=0$ and $c_{1}=1 / a_{v}$. Conceptually, self 0 knows how to compute the equilibrium and knows $\left(c_{0}, c_{1}, \alpha\right)$ but cannot impose a demand function on self 1 . Self 1 
is boundedly rational and adds receiver noise to the best price signal. Thus, investor $i$ cannot costlessly process the price information, and she can only read a coarser signal as follows:

$$
\tilde{s}_{p, i}=\left(c_{0}+\frac{1}{\sqrt{\tau_{u, i}}} \tilde{u}+\frac{1}{\sqrt{\tau_{e, i}}} \tilde{e}_{i}\right)+c_{1} \tilde{p}=\tilde{v}+\alpha \tilde{u}+\frac{1}{\sqrt{\tau_{u, i}}} \tilde{u}+\frac{1}{\sqrt{\tau_{e, i}}} \tilde{e}_{i} .
$$

Using Bayes' rule, we can compute

$$
\begin{aligned}
E\left(\tilde{v} \mid \tilde{s}_{i}, \tilde{s}_{p, i}\right) & =\left(\tau_{\varepsilon} \tilde{s}_{i}+\frac{\tau_{e, i} \tau_{u, i}}{\tau_{u, i}+\tau_{e, i}\left(\alpha \sqrt{\tau_{u, i}}+1\right)^{2}} \tilde{s}_{p, i}\right) \operatorname{Var}\left(\tilde{v} \mid \tilde{s}_{i}, \tilde{s}_{p, i}\right), \\
\operatorname{Var}\left(\tilde{v} \mid \tilde{s}_{i}, \tilde{s}_{p, i}\right) & =\frac{\tau_{u, i}+\tau_{e, i}\left(\alpha \sqrt{\tau_{u, i}}+1\right)^{2}}{\tau_{e, i} \tau_{u, i}+\left[\tau_{u, i}+\tau_{e, i}\left(\alpha \sqrt{\tau_{u, i}}+1\right)^{2}\right]\left(\tau_{v}+\tau_{\varepsilon}\right)}
\end{aligned}
$$

Inserting these two expressions into (5), we can compute the expression of $D\left(\tilde{p} ; \tilde{s}_{i}, \tilde{s}_{p, i}\right)$, which is in turn inserted into (6) to compute the equilibrium price as a function of $\tilde{v}$ and $\tilde{u}$. Comparing coefficients with the conjectured price function (8), we can form a system of equations to determine the two unknown price coefficients $a_{v}$ and $a_{u}$.

Proposition 1 (Financial market equilibrium) Suppose that investors have the same sophistication level (i.e., $\tau_{u, i}=\tau_{u}$ and $\tau_{e, i}=\tau_{e}$, for $i \in[0,1]$ ). There exists a unique linear equilibrium price function,

$$
\tilde{p}=a_{v} \tilde{v}+a_{u} \tilde{u}
$$

where

$$
\begin{aligned}
a_{v} & =\frac{\tau_{e} \tau_{u}+\tau_{\varepsilon}\left[\tau_{u}+\tau_{e}\left(\alpha \sqrt{\tau_{u}}+1\right)^{2}\right]}{\tau_{e} \tau_{u}+\left[\tau_{u}+\tau_{e}\left(\alpha \sqrt{\tau_{u}}+1\right)^{2}\right]\left(\tau_{v}+\tau_{\varepsilon}\right)}, \\
a_{u} & =\frac{\tau_{e} \sqrt{\tau_{u}}\left(\alpha \sqrt{\tau_{u}}+1\right)}{\tau_{e} \tau_{u}+\left[\tau_{u}+\tau_{e}\left(\alpha \sqrt{\tau_{u}}+1\right)^{2}\right]\left(\tau_{v}+\tau_{\varepsilon}\right)}
\end{aligned}
$$

where $\alpha \equiv \frac{a_{u}}{a_{v}} \in\left(0, \frac{\tau_{e} \sqrt{\tau_{u}}}{\tau_{e} \tau_{\varepsilon}+\tau_{u} \tau_{\varepsilon}}\right)$ is uniquely determined by the positive real root of the following cubic equation:

$$
\tau_{e} \tau_{u} \tau_{\varepsilon} \alpha^{3}+2 \tau_{e} \sqrt{\tau_{u}} \tau_{\varepsilon} \alpha^{2}+\left(\tau_{e} \tau_{\varepsilon}+\tau_{u} \tau_{\varepsilon}\right) \alpha-\tau_{e} \sqrt{\tau_{u}}=0
$$

Costly interpretation of asset prices brings an endogenous noise $\tilde{u}$ into the price system. Even 
if investors were to get rid of the noise $\tilde{u}$ in the price system by setting $\tau_{u} \rightarrow \infty$, investors would not still be fully sophisticated and thus they would not be able to extract the best signal from the price if $\tau_{e}<\infty$. In the limiting case in which both $\tau_{u} \rightarrow \infty$ and $\tau_{e} \rightarrow \infty$, we have $\tilde{s}_{p, i}=\tilde{s}_{p}$, and the noise $\tilde{u}$ vanishes in the price function, making the economy degenerate into the full REE setting.

Corollary 1 Suppose $\tau_{u, i}=\tau_{u}$ and $\tau_{e, i}=\tau_{e}$, for $i \in[0,1]$. Given $\left(\tau_{v}, \tau_{\varepsilon}\right) \in \mathbb{R}_{++}^{2}$ :

1. Fix $\tau_{e} \in(0, \infty)$, as $\tau_{u} \rightarrow \infty$, then $a_{v}=\frac{\tau_{e}+\tau_{\varepsilon}}{\tau_{e}+\tau_{v}+\tau_{\varepsilon}}$ and $a_{u}=0$;

2. Fix $\tau_{u} \in(0, \infty)$, as $\tau_{e} \rightarrow \infty$, then $a_{v}=\frac{\tau_{u}+\tau_{\varepsilon}\left(\alpha \sqrt{\tau_{u}}+1\right)^{2}}{\tau_{u}+\left(\alpha \sqrt{\tau_{u}}+1\right)^{2}\left(\tau_{v}+\tau_{\varepsilon}\right)}$ and $a_{u}=\frac{\sqrt{\tau_{u}}\left(\alpha \sqrt{\tau_{u}}+1\right)}{\tau_{u}+\left(\alpha \sqrt{\tau_{u}}+1\right)^{2}\left(\tau_{v}+\tau_{\varepsilon}\right)}$;

3. As both $\tau_{u} \rightarrow \infty$ and $\tau_{e} \rightarrow \infty$, the price function converges almost surely to $\tilde{p}^{R E E}=\tilde{v}$ and $\tilde{s}_{p, i}=\tilde{s}_{p}$.

\subsection{Price Momentum}

We now present one main result of our model — price momentum — which says that the current price $\tilde{p}$ positively predicts asset returns $\tilde{v}-\tilde{p}$. Empirically, one can run a linear regression from $\tilde{v}-\tilde{p}$ on $\tilde{p}$, i.e., $\tilde{v}-\tilde{p}=$ intercept $+m \times \tilde{p}+$ error. The regression coefficient is $m=\frac{\operatorname{Cov}(\tilde{v}-\tilde{p}, \tilde{p})}{\operatorname{Var}(\tilde{p})}$. In the traditional noisy-REE setting with exogenous noise trading (e.g., Hellwig, 1980), returns exhibit reversals; that is, $m<0$ (see Banerjee et al., 2009). This is because exogenous noise demand pushes the price too high and exogenous noisy supply depresses the price too low. In contrast, in our setting with endogenous noise trading due to costly interpretation of prices, returns exhibit momentum: $m>0$. This result provides an explanation for the price momentum documented in the data (e.g., Jegadeesh and Titman, 1993; Moskowitz et al., 2012).

The price momentum in our model is an underreaction story. When investors are fully sophisticated $\left(\tilde{s}_{p, i}=\tilde{s}_{p}\right)$, the price fully aggregates their private information and there is no return predictability. Formally, by Part 3 of Corollary 1, the price is a martingale $\left(\tilde{p}^{R E E}=E\left(\tilde{v} \mid \tilde{p}^{R E E}\right)\right)$ and hence the price change is not predictable $\left(\operatorname{Cov}\left(\tilde{v}-\tilde{p}^{R E E}, \tilde{p}^{R E E}\right)=0\right)$. When investors have limited sophistication, their forecasts do not fully use the information in the price, which in turn 
causes their trading not to fully incorporate information, thereby making the price underreact to information. Our mechanism shares similarity to Hong and Stein (1999) and Eyster et al. (2019) who generate momentum via traders that make forecasts based on private information, but do not fully infer the information from prices. Since these investors fail to extract information from prices, the slow diffusion generates momentum.

Proposition 2 (Return Predictiveness) Returns exhibit price momentum:

$$
m=\frac{\operatorname{Cov}(\tilde{v}-\tilde{p}, \tilde{p})}{\operatorname{Var}(\tilde{p})}>0
$$

\subsection{Implications of Investor Sophistication}

In this subsection, we examine how investor sophistication affects asset prices and trading volume. We assume that all investors have a common sophistication level $\tau_{u, i}=\tau_{u}$ and $\tau_{e, i}=\tau_{e}$ and conduct comparative static analysis with respect to $\tau_{u}$ and $\tau_{e}$. In a full equilibrium setting, $\tau_{u, i}$ and $\tau_{e, i}$ will be determined endogenously, which will be explored later in Section 5 .

\subsubsection{Sophistication Level $\tau_{u}$ of Taming Sentiment}

The parameter values in Figure 1 follow from the calibration exercise conducted by Kovalenkov and Vives (2014). Specifically, we interpret one period as one year and let the total asset payoff volatility match its historical value $20 \%$ of the aggregate stock market (i.e., $\sqrt{\operatorname{Var}(\tilde{v})}=20 \%$ ). Regarding private information quality, Kovalenkov and Vives (2014) consider a range of signalto-noise ratio $\frac{\tau_{\varepsilon}}{\tau_{v}}$, from as low as one basis point to as high as 16 . We assume $\frac{\tau_{\varepsilon}}{\tau_{v}}=1$. We also follow Kovalenkov and Vives (2014) and set the risk aversion coefficient $\gamma$ at 2. Finally, we assume that the sophistication level of curbing the individual processing error is constant at $\tau_{e}=500$, which implies that investors make very small errors in forming their date-1 beliefs

(i.e., $\left.\operatorname{Var}\left(\frac{1}{\sqrt{\tau_{e}}} \tilde{e}_{i}\right)=\frac{1}{\tau_{e}}=0.2 \%\right)$. We do so deliberately to illustrate that even small errors in interpreting price information can aggregate into a significant effect on equilibrium outcomes. 
Figure 1: Implications of $\tau_{u}$ for Asset Prices and Trading Volume
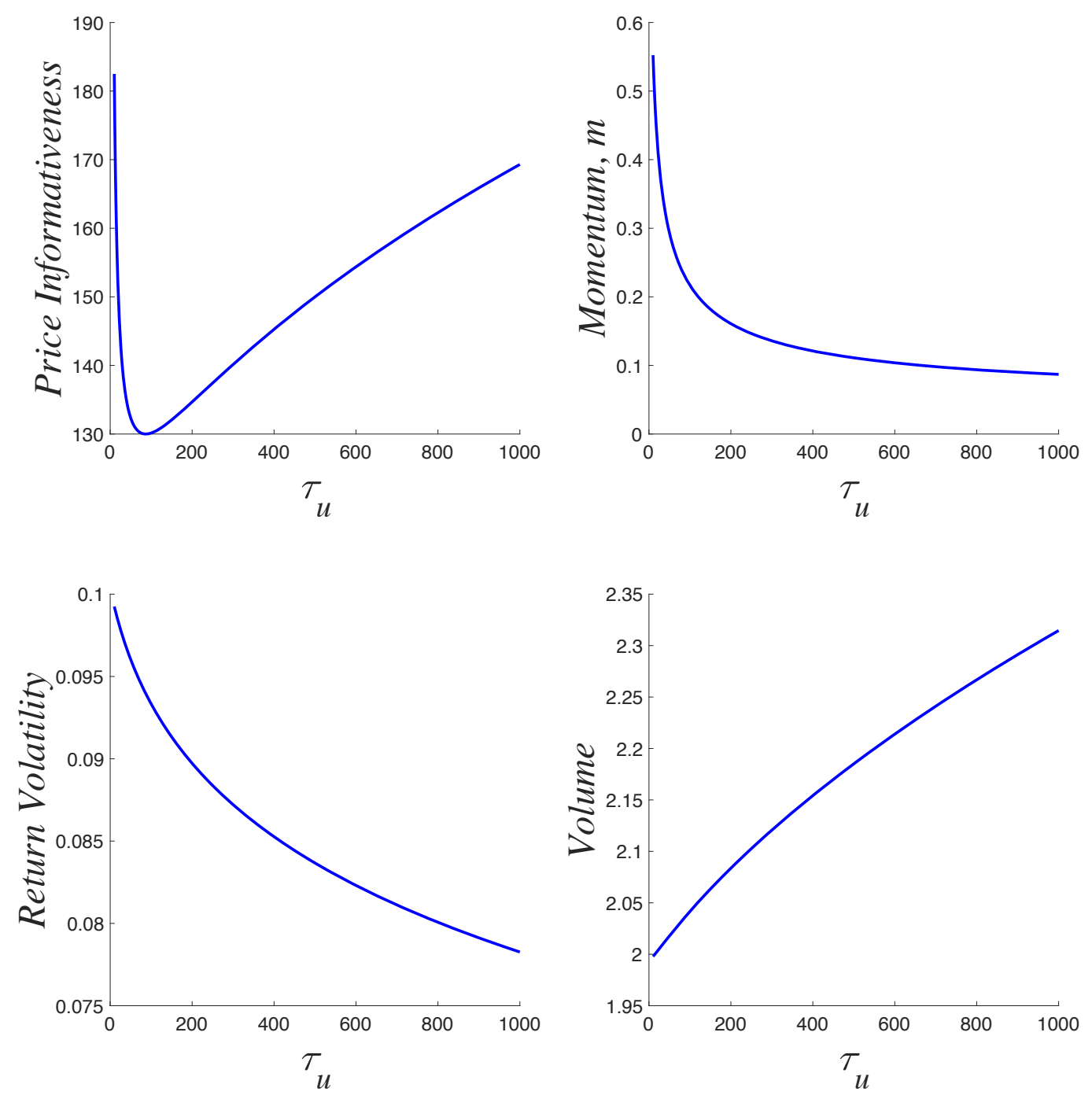

This figure plots price informativeness $\left(\frac{1}{\operatorname{Var}(\tilde{v} \mid \tilde{p})}\right)$, return volatility $(\sigma(\tilde{v}-\tilde{p}))$, price momentum $(m)$, and trading volume against the investor sophistication $\tau_{u}$ of taming the sentiment error. The parameters are set as follows: $\tau_{v}=\tau_{\varepsilon}=25, \tau_{e}=500$ and $\gamma=2$.

Price informativeness As standard in the literature (e.g., Vives, 2008; Peress, 2010), we use the precision $\frac{1}{\operatorname{Var}(\tilde{v} \mid \tilde{p})}$ of stock payoff conditional on its price to measure "price informativeness" (or "market efficiency," "informational efficiency," and "price efficiency"). By equation (8), applying Bayes' rule delivers $\frac{1}{\operatorname{Var}(\tilde{v} \tilde{p})}=\tau_{v}+\frac{1}{\alpha^{2}}$. Since $\tau_{v}$ is an exogenous constant, price informativeness is negatively related to $\alpha$.

In the top left panel of Figure 1, we plot price informativeness $\frac{1}{\operatorname{Var}(\tilde{v} \tilde{p})}$ against the investor 
sophistication $\tau_{u}$ of taming the sentiment error. When $\tau_{u}=0$ or $\tau_{e}=0$, we have $\alpha=0$ and $\frac{1}{\operatorname{Var}(\tilde{v} \mid \tilde{p})}=\infty$. When either of these two cases occurs, traders do not observe a price signal. Hence, common noise $u$ does not get incorporated into the price so that $a_{u}=0$ and $a_{v}=\frac{\tau_{\varepsilon}}{\tau_{v}+\tau_{\varepsilon}}$, which implies that $\alpha=0$ and $\frac{1}{\operatorname{Var}(\tilde{v} \mid \tilde{p})}=\infty$. Also, as a comparison, the $\alpha$ value in the standard REE economy with both $\tau_{u} \rightarrow \infty$ and $\tau_{e} \rightarrow \infty$ is zero. We observe (a) that costly interpretation of prices injects noise into the price as long as investors are not fully sophisticated (i.e., $\alpha>0$ and $\frac{1}{\operatorname{Var}(\tilde{v} \mid \tilde{p})}<\infty$ for $\left.\tau_{u} \in(0, \infty)\right)$; and $(\mathrm{b})$ that price informativeness $\frac{1}{\operatorname{Var}(\tilde{v} \mid \tilde{p})}$ is non-monotonic in sophistication $\tau_{u}$. Intuitively, if $\tau_{u}$ increases from 0 to some positive value, trading injects noise $u$ into the price, lowering informativeness. However, if $\tau_{u}$ continues to increase further to infinity, then the price becomes a more precise signal. This price-informativeness result has important implications for determining the sophistication level in Section 5.

Return volatility Return volatility is measured by the standard deviation of asset returns, $\sigma(\tilde{v}-\tilde{p})$. In the bottom left panel of Figure 1, we plot return volatility $\sigma(\tilde{v}-\tilde{p})$ against the investor sophistication $\tau_{u}$ of taming the sentiment error. Return volatility in the standard REE economy with both $\tau_{u} \rightarrow \infty$ and $\tau_{e} \rightarrow \infty$ is zero. Costly interpretation of prices generates higher return volatility than the full REE benchmark. This may help to address the volatility puzzle (Shiller, 1981; LeRoy and Porter, 1981), which states that it is difficult to explain the historical volatility of stock returns with any model in which investors are rational and discount rates are constant. Also note that the excess return volatility is non-negligible even though investors only make very small mistakes.

Return predictability In Proposition 2, we have shown that our model generates price momentum $m>0$. In the top right panel of Figure 1, we plot $m$ against the investor sophistication $\tau_{u}$ of taming the sentiment error. Return predictability in the standard REE economy with both $\tau_{u} \rightarrow \infty$ and $\tau_{e} \rightarrow \infty$ is zero. We observe that, consistent with Proposition $2, m$ is indeed positive, indicating that there exists price momentum in our economy. In addition, $m$ is decreasing in $\tau_{u}$ as the information obtained from the price signal is becoming more precise. 
Trading volume In the bottom right panel of Figure 1, we plot trading volume $\int_{0}^{1}\left|D\left(p ; s_{i}, s_{p, i}\right)\right| d i$ against $\tau_{u}$. Trading volume in the standard REE economy with both $\tau_{u} \rightarrow \infty$ and $\tau_{e} \rightarrow \infty$ is zero. We see that costly price interpretation generates excess trading volume. Trading volume in the model is positive since traders have private information and do not fully learn all the information about fundamentals from prices. In our numerical exercise, trading volume is increasing in $\tau_{u}$. Intuitively, a high $\tau_{u}$ decreases the weight of the common sentiment component $\tilde{u}$ in the price signal, which indirectly makes the individual processing error $\tilde{e}_{i}$ relatively more important in the price signal. Hence, there is an increase in disagreement and an increase in volume as a result of an increase in $\tau_{u}$.

Proposition 3 (Implications of $\tau_{u}$ )

(a) Price informativeness

(1) For sufficiently small $\tau_{u}$, as investors become more sophisticated by increasing $\tau_{u}$, price $\tilde{p}$ conveys less precise information about fundamental $\tilde{v}$.

(b) Return volatility

(1) As $\tau_{u} \rightarrow \infty$, return volatility approaches $\sqrt{\frac{\tau_{v}}{\left(\tau_{e}+\tau_{v}+\tau_{\varepsilon}\right)^{2}}}$.

(2) As $\tau_{u} \rightarrow 0$, return volatility approaches $\sqrt{\frac{\tau_{e}^{2} \tau_{v}}{\left(\tau_{e} \tau_{v}+\tau_{e} \tau_{\varepsilon}\right)^{2}}}$.

(c) Trading Volume

(1) As $\tau_{u} \rightarrow \infty$, the total trading volume does not vanish (i.e., $\lim _{\tau_{u} \rightarrow \infty}$ Volume $>0$ ).

(2) As $\tau_{u} \rightarrow 0$, the total trading volume does not vanish (i.e., $\lim _{\tau_{u} \rightarrow \infty}$ Volume $>0$ ).

\subsubsection{Sophistication Level $\tau_{e}$ of Curbing Individual Processing Error}

In Figure 2, we analyze the effects of changes in $\tau_{e}$ on asset prices and trading volume. The parameter values in Figure 2 are the same as Figure 1 with the exception that the sophistication level of taming sentiment is now fixed at $\tau_{u}=500$, which implies small sentiment-driven errors in self-1 beliefs (i.e., $\operatorname{Var}\left(\frac{1}{\sqrt{\tau_{u}}} \tilde{u}_{i}\right)=\frac{1}{\tau_{u}}=0.2 \%$ ). 
Figure 2: Implications of $\tau_{e}$ for Asset Prices and Trading Volume
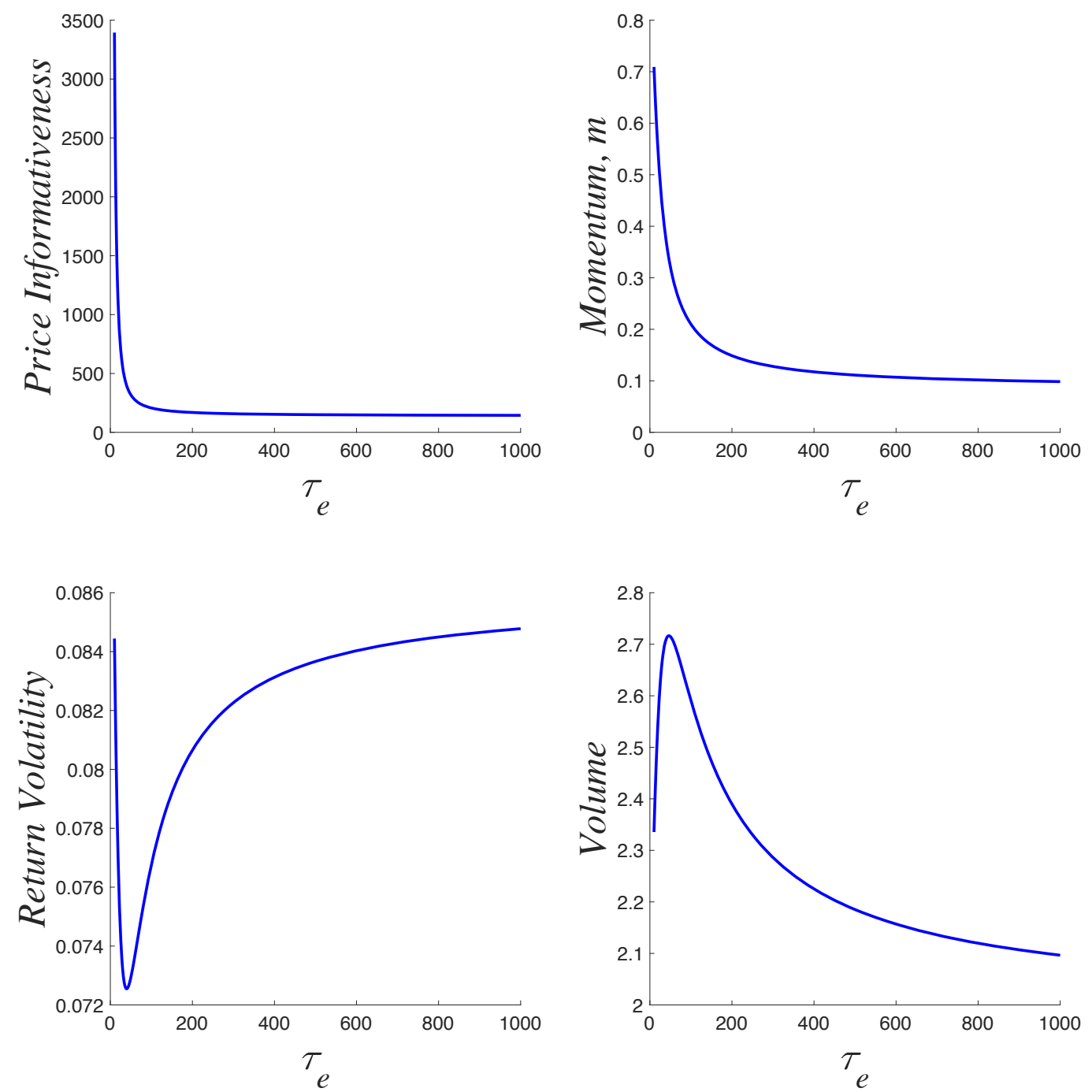

This figure plots price informativeness $\left(\frac{1}{\operatorname{Var}(\tilde{v} \mid \tilde{p})}\right)$, return volatility $(\sigma(\tilde{v}-\tilde{p}))$, price momentum $(m)$, and trading volume against the investor sophistication $\tau_{e}$ of curbing individual processing error. The parameters are set as follows: $\tau_{v}=\tau_{\varepsilon}=25, \tau_{u}=500$ and $\gamma=2$.

Price informativeness In the top left panel of Figure 2, we plot price informativeness $\frac{1}{\operatorname{Var}(\tilde{v} \mid \tilde{p})}$ against the investor sophistication $\tau_{e}$ of curbing individual processing errors. As a comparison, the $\alpha$ value in the standard REE economy with both $\tau_{u} \rightarrow \infty$ and $\tau_{e} \rightarrow \infty$ is zero, and hence $\frac{1}{\operatorname{Var}(\tilde{v} \tilde{p} \tilde{p})}=\tau_{v}+\frac{1}{\alpha^{2}}=\infty$. Price informativeness is monotonically decreasing in $\tau_{e}$. Intuitively, at $\tau_{e}=0$, the price is extremely noisy and investors completely disregard the price signal. Hence, the price is fully revealing. As $\tau_{e}$ increases, investors start using the price signal and the sentiment 
noise $\tilde{u}$ is added to the price. At the same time, as $\tau_{e}$ increases, the weight of sentiment $\tilde{u}$ in the individual price signal increases relative to the weight of the individual processing error, making the signal more dependent on sentiment and hence the price more noisy.

Return volatility In the bottom left panel of Figure 2, we plot return volatility $\sigma(\tilde{v}-\tilde{p})$ against the investor sophistication $\tau_{e}$ of curbing individual processing errors. Return volatility in the standard REE economy is zero. Return volatility is non-monotonic in $\tau_{e}$. As $\tau_{e}$ increases, (a) traders have a more precise individual price signal, bringing the price closer to fundamentals and reducing volatility, and (b) the weight of sentiment $\tilde{u}$ in the individual price signal increases relative to the weight of the individual processing error, making the signal more dependent on sentiment and hence the price more noisy and further away from fundamentals.

Return predictability In Proposition 2, we have shown that our model generates momentum, i.e., $m>0$. In the top right panel of Figure 2, we plot $m$ against $\tau_{e}$. Return predictability in the standard REE economy is zero. We observe that $m$ is indeed positive, indicating that there exists price momentum in the economy. In addition, $m$ is decreasing in $\tau_{e}$ as the information obtained from the price signal is becoming more precise.

Trading volume In the bottom right panel of Figure 2, we plot trading volume against $\tau_{e}$. Trading volume in the standard REE economy is zero. We see that costly price interpretation generates trading volume since prices are not fully revealing. In our numerical exercise, trading volume is non-monotonic in $\tau_{e}$. Intuitively, an increase in $\tau_{e}$ from $\tau_{e}=0$ provides traders with a price signal, which is an additional source of disagreement between investors, leading to an increase in trading volume. However, as $\tau_{e}$ increases even further the disagreement diminishes and trading volume decreases as well.

Proposition 4 (Implications of $\tau_{e}$ )

\section{(a) Price informativeness}


(1) As investors become more sophisticated by increasing $\tau_{e}$, price $\tilde{p}$ conveys less precise information about fundamental $\tilde{v}$.

(b) Return volatility

(1) As $\tau_{e} \rightarrow \infty$, return volatility approaches

$$
\sigma(\tilde{v}-\tilde{p})=\sqrt{\frac{\left(\alpha \sqrt{\tau_{u}}+1\right)^{2}\left(\tau_{v} \alpha^{2} \tau_{u}+2 \tau_{v} \alpha \sqrt{\tau_{u}}+\tau_{u}+\tau_{v}\right)}{\left(\tau_{v}+\tau_{\varepsilon}+\tau_{u}+\alpha^{2} \tau_{u} \tau_{v}+\alpha^{2} \tau_{u} \tau_{\varepsilon}+2 \alpha \sqrt{\tau_{u}} \tau_{v}+2 \alpha \sqrt{\tau_{u}} \tau_{\varepsilon}\right)^{2}}} .
$$

(2) As $\tau_{e} \rightarrow 0$, return volatility approaches $\sqrt{\frac{\tau_{u}^{2} \tau_{v}}{\left(\tau_{u} \tau_{v}+\tau_{u} \tau_{\varepsilon}\right)^{2}}}$.

(c) Trading Volume

(1) As $\tau_{e} \rightarrow \infty$, the total trading volume does not vanish (i.e., $\lim _{\tau_{e} \rightarrow \infty}$ Volume $>0$ ).

(2) As $\tau_{e} \rightarrow 0$, the total trading volume does not vanish (i.e., $\lim _{\tau_{e} \rightarrow 0}$ Volume $>0$ ).

\section{Sophistication Level Equilibrium}

\subsection{Sophistication Determination}

As we discussed in Section 3.1, the sophistication levels are determined by the rational self 0 of each investor at date 0 . Inserting the date-1 demand function $D\left(\tilde{p} ; \tilde{s}_{i}, \tilde{s}_{p, i}\right)$ in $(5)$ into the CARA utility function and taking expectations yield investor $i$ 's date-0 payoff, $E\left[-e^{-\gamma\left[(\tilde{v}-\tilde{p}) D\left(\tilde{p} ; \tilde{s}_{i}, \tilde{s}_{p, i}\right)-C\left(\tau_{u, i}, \tau_{e, i}\right)\right]}\right]$. Note that this expectation is computed under the correct distribution, because self 0 is fully rational in contemplating the sophistication levels $\tau_{u, i}$ and $\tau_{e, i}$ of her future self, which in turn determine how much information the boundedly rational self 1 will read from the asset price $\tilde{p}$. Formally, trader $i$ chooses $\tau_{u, i}$ and $\tau_{e, i}$ to maximize

$$
\max _{\left(\tau_{u, i}, \tau_{e, i}\right)} E\left[-e^{-\gamma\left[(\tilde{v}-\tilde{p}) D\left(\tilde{p} ; \tilde{s}_{i}, \tilde{s}_{p, i}\right)-C\left(\tau_{u, i}, \tau_{e, i}\right)\right]}\right] .
$$

Definition 1 An overall equilibrium of the two-stage game is defined as follows: 
(a) Financial market equilibrium at date 1 , which is characterized by a price function $p(\tilde{v}, \tilde{u})$ and demand functions $D\left(\tilde{p} ; \tilde{s}_{i}, \tilde{s}_{p, i}\right)$, such that:

(1) $D\left(\tilde{p} ; \tilde{s}_{i}, \tilde{s}_{p, i}\right)$ is given by (5), which maximizes investors' conditional subjective expected utilities given their date-1 beliefs;

(2) the market clears almost surely, i.e., equation (6) holds;

(3) investors' date-1 beliefs are given by (11) and (12), where $\tilde{s}_{p}$ is implied by the equilibrium price function $p(\tilde{v}, \tilde{u})$ and where the sophistication levels $\left(\tau_{u, i}\right)_{i \in[0,1]}$ and $\left(\tau_{e, i}\right)_{i \in[0,1]}$ are determined at date 0 .

(b) Sophistication level equilibrium at date 0, which is characterized by sophistication levels $\left(\tau_{u, i}\right)_{i \in[0,1]}$ and $\left(\tau_{e, i}\right)_{i \in[0,1]}$, such that $\left(\tau_{u, i}, \tau_{e, i}\right)$ solves (14), where investors' date-1 beliefs are given by (11) and (12).

\subsection{Equilibrium Characterization}

Given that investors are ex ante identical, we consider a symmetric equilibrium in which all investors choose the same sophistication level. Let $W\left(\tau_{u, i}, \tau_{e, i} ; \tau_{u}, \tau_{e}\right)$ denote the certainty equivalent of investor $i$ 's date- 0 payoff when she decides to acquire sophistication levels $\tau_{u, i}$ and $\tau_{e, i}$ and all the other investors acquire sophistication levels $\tau_{u}$ and $\tau_{e}$. That is,

$$
\begin{gathered}
W\left(\tau_{u, i}, \tau_{e, i} ; \tau_{u}, \tau_{e}\right) \equiv-\frac{1}{\gamma} \ln \left(-E\left[-e^{-\gamma\left[(\tilde{v}-\tilde{p}) D\left(\tilde{p}, \tilde{s}_{i}, \tilde{s}_{p, i}\right)-C\left(\tau_{u, i}, \tau_{e, i}\right)\right]}\right]\right) \\
=\frac{1}{2 \gamma} \ln \left[\begin{array}{c}
{[1+\gamma \underbrace{\operatorname{Cov}\left(\tilde{v}-\tilde{p}, D_{i}\right)}_{\text {more informed trading }}]^{2}} \\
-\gamma^{2} \operatorname{Var}(\tilde{v}-\tilde{p}) \times \underbrace{\operatorname{Var}\left(D_{i}\right)}_{\text {excessive trading }}
\end{array}\right]-\underbrace{C\left(\tau_{u, i}, \tau_{e, i}\right)}_{\text {cost }},
\end{gathered}
$$

where the second equality follows from the properties of normal distributions.

In equation (15), improving the sophistication of future self 1 affects the current self 0 's payoff in three ways. The first effect is a direct effect: acquiring sophistication incurs a cost, 
$C\left(\tau_{u, i}, \tau_{e, i}\right)$, which directly harms the investor from self 0's perspective. The other two effects are indirect, which work through affecting the trading in the future financial market. These two indirect effects work in opposite directions.

First, being more sophisticated allows the future self 1 to better read information from the asset price, which therefore makes her trading more aligned with price changes - i.e., buying low and selling high - and therefore benefits the investor at date 0 . This positive indirect effect is captured by the term $\operatorname{Cov}\left(\tilde{v}-\tilde{p}, D_{i}\right)$. Intuitively, when $\tau_{u, i}>\tau_{u}$ and $\tau_{e, i}>\tau_{e}$, investor $i$ 's forecast beats the market and thus, her trading improves her ex-ante welfare.

Second, investors engage in speculative trading in the date-1 financial market, because there are no risk-sharing benefits in our setting. In this sense, trading is excessive, and the more an investor's future self trades, the more harmful it is from self 0's perspective. Improving the sophistication levels $\tau_{u, i}>\tau_{u}$ and $\tau_{e, i}>\tau_{e}$ allows self 1 to lower her perceived risk and thus she will trade more aggressively, which in turn harms self 0 via the excessive trading channel. This negative effect is captured by the term $\operatorname{Var}\left(D_{i}\right)$ in equation (15), which measures the size of self 1 's trading in the financial market.

The optimal sophistication levels $\tau_{u, i}^{*}$ and $\tau_{e, i}^{*}$ are determined by the first-order conditions (FOCs) of maximizing $W\left(\tau_{u, i}, \tau_{e, i} ; \tau_{u}, \tau_{e}\right)$. Formally, $\left(\tau_{u, i}^{*}, \tau_{e, i}^{*}\right)=\arg \max _{\tau_{u, i}, \tau_{e, i}} W\left(\tau_{u, i}, \tau_{e, i} ; \tau_{u}^{*}, \tau_{e}^{*}\right)$. The FOCs of the sophistication determination problem are given by

$$
\left\{\begin{array}{cc}
\left.\frac{\partial W\left(\tau_{u, i}, \tau_{e, i} ; \tau_{u}, \tau_{e}\right)}{\partial \tau_{u, i}}\right|_{\tau_{u, i}=0} \leq 0, & \text { if } \tau_{u, i}=0 \\
\frac{\partial W\left(\tau_{u, i}, \tau_{e, i} ; \tau_{u}, \tau_{e}\right)}{\partial \tau_{u, i}}=0, & \text { if } \tau_{u, i}>0
\end{array}\right.
$$

and

$$
\left\{\begin{array}{cc}
\left.\frac{\partial W\left(\tau_{u, i}, \tau_{e, i} ; \tau_{u}, \tau_{e}\right)}{\partial \tau_{e, i}}\right|_{\tau_{e, i}=0} \leq 0, & \text { if } \tau_{e, i}=0, \\
\frac{\partial W\left(\tau_{u, i}, \tau_{e, i} ; \tau_{u}, \tau_{e}\right)}{\partial \tau_{e, i}}=0, & \text { if } \tau_{e, i}>0 .
\end{array}\right.
$$

In a symmetric equilibrium, we impose $\tau_{u, i}^{*}=\tau_{u}^{*}$ and $\tau_{e, i}^{*}=\tau_{e}^{*}$ in the FOCs. The overall equilibrium is characterized by three variables, $\left(\tau_{u}^{*}, \tau_{e}^{*}, \alpha^{*}\right)$. These three variables are jointly determined by a system of three conditions: (a) the cubic equation (13) characterizing the 
financial market equilibrium; (b) the FOC (16) characterizing the optimal sophistication level $\tau_{u}^{*}$ of taming the sentiment error; and (c) the FOC (17) characterizing the optimal sophistication level $\tau_{e}^{*}$ of curbing individual processing errors.

The complexity of the problem precludes a full analytical characterization of the equilibrium. We therefore rely on numerical analysis. We consider a linear cost function, $C\left(\tau_{u, i}, \tau_{e, i}\right)=$ $\kappa_{u} \tau_{u, i}+\kappa_{e} \tau_{e, i}$, where $\kappa_{u}$ and $\kappa_{e}$ are positive constants. ${ }^{7}$ Our model admits multiple equilibria. We use Figure 3 to plot the first equilibrium and Figure 4 to plot the second equilibrium. In Panels a1-a3 of both figures, we plot the equilibrium values of $\left(\tau_{u}^{*}, \tau_{e}^{*}, \alpha^{*}\right)$ as functions of $\kappa_{u}$, when $\kappa_{e}$ is fixed at 0.0002 . In Panels b1-b3 of both figures, we vary $\kappa_{e}$ and fix the value of $\kappa_{u}$ at 0.0015. The other parameters take the same values as in previous figures, that is, $\tau_{v}=25$ and $\gamma=2$, except for $\tau_{\varepsilon}=1$. We set a smaller $\tau_{\varepsilon}$ because under coarse private information, there are multiple equilibria at the sophistication determination stage. Numerically, we have only been able to find two equilibria.

The qualitative patterns of $\left(\tau_{u}^{*}, \tau_{e}^{*}, \alpha^{*}\right)$ are similar across both figures and thus we focus on Figure 3. Intuitively, an increase in the cost $\kappa_{u}$ of taming the sentiment error leads to a decrease in both sophistication levels $\tau_{u}^{*}$ and $\tau_{e}^{*}$ in Panels a1-a2, and an increase in the cost $\kappa_{e}$ of curbing individual processing errors leads to a decrease in both $\tau_{u}^{*}$ and $\tau_{e}^{*}$ in Panels b1-b2. However, Panels a3 and b3 exhibit different patterns of of $\alpha^{*}$. Specifically, in Panel a3, $\alpha^{*}$ is non-monotonic in $\kappa_{u}$, while in Panel b3, $\alpha^{*}$ is monotonically decreasing in $\kappa_{e}$. This difference is caused by the different behavior of $\tau_{u}^{*}$ : In Panel a1, changes in $\kappa_{u}$ have a big impact on the choice of $\tau_{u}^{*}$, and as we have seen in Figure 1, price informativeness is non-monotonic; by contrast, changes in $\kappa_{e}$ only causes modest variations in $\tau_{u}^{*}$ in Panel b1, and hence price informativeness is monotonic.

\subsection{What Drives Equilibrium Multiplicity?}

In this subsection, we dig deeper the driving forces of multiple equilibria by distinguishing the choice of sophistication $\tau_{u, i}$ of taming the sentiment error from the choice of sophistication $\tau_{e, i}$

\footnotetext{
${ }^{7}$ We use a linear and separable cost function because it serves as a simple benchmark to understand the numerical results.
} 
Figure 3: The First Overall Equilibrium

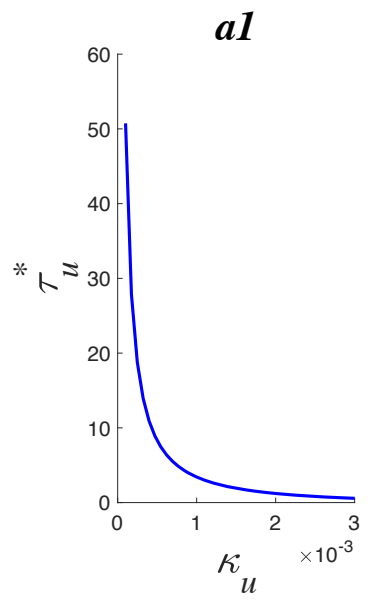

b1

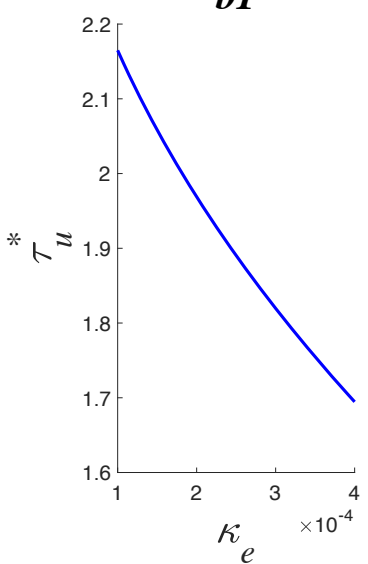

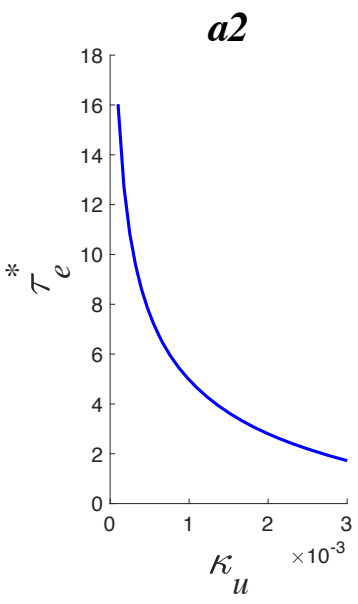

b2

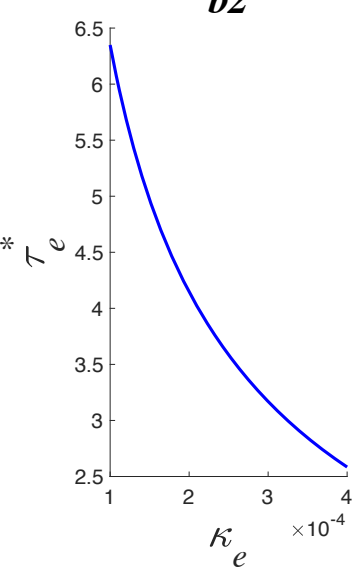

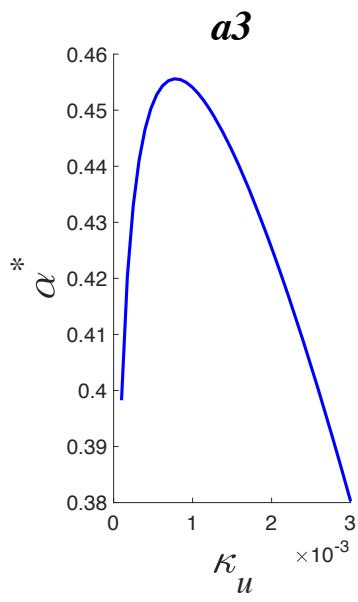

b3

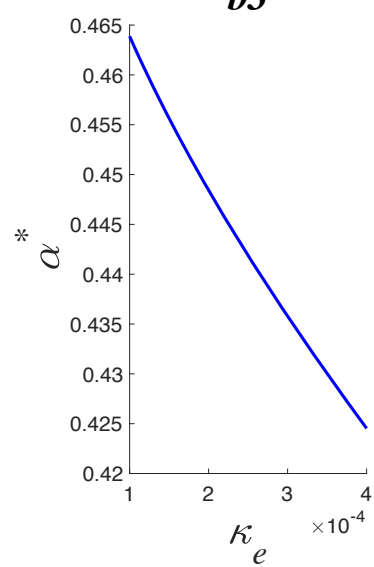

This figure plots values of $\left(\tau_{u}^{*}, \tau_{e}^{*}, \alpha^{*}\right)$ for the first equilibrium as functions of $\kappa_{u}$ in the top panels and $\kappa_{e}$ in the bottom panels. The cost function is $C\left(\tau_{u, i}, \tau_{e, i}\right)=\kappa_{u} \tau_{u, i}+\kappa_{e} \tau_{e, i}$, where $\kappa_{u}$ and $\kappa_{e}$ are positive constants. The parameters are set as follows: $\tau_{v}=25, \tau_{\varepsilon}=1$ and $\gamma=2$.

of curbing individual processing errors.

The Choice of $\tau_{u}$ We now take $\tau_{e, i}=\tau_{e}$ as given and focus on the choice of $\tau_{u, i}$. Since the cost function $C\left(\tau_{u, i}, \tau_{e, i}\right)$ is exogenous, we focus on analyzing the marginal benefit and simply set $C\left(\tau_{u, i}, \tau_{e, i}\right)=0$. Let us denote the marginal benefit of choosing $\tau_{u, i}=\tau_{u}$ as $\phi\left(\tau_{u}\right)$. The shape of $\phi\left(\tau_{u}\right)=\left.\frac{\partial W\left(\tau_{u, i}, \tau_{u}\right)}{\partial \tau_{u, i}}\right|_{\tau_{u, i}=\tau_{u}}$ will determine whether there is a unique equilibrium or there are multiple equilibria. Formally, if $\phi\left(\tau_{u}\right)$ is downward sloping, then the equilibrium is unique. In contrast, when $\phi\left(\tau_{u}\right)$ has an upward sloping segment, multiplicity can arise. Hence, the key to have multiple equilibria is to check the sign of $\frac{d \phi\left(\tau_{u}\right)}{d \tau_{u}}$. 
Figure 4: The Second Overall Equilibrium

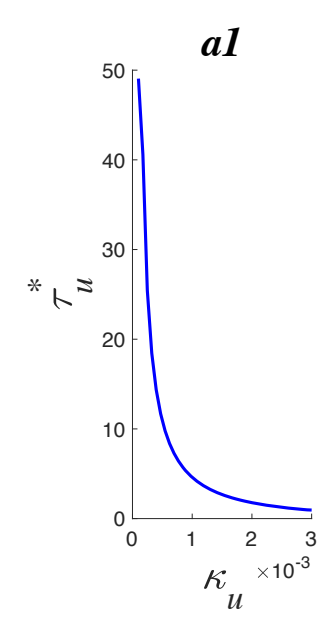

b1

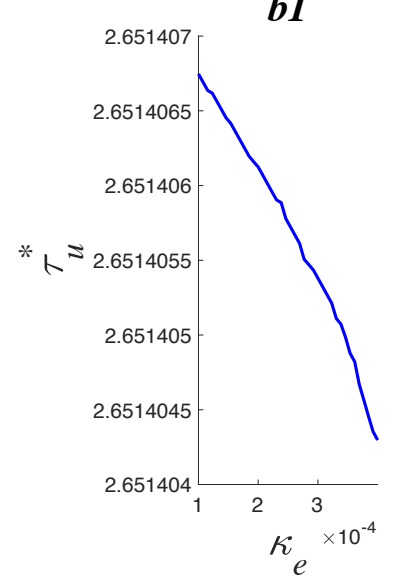

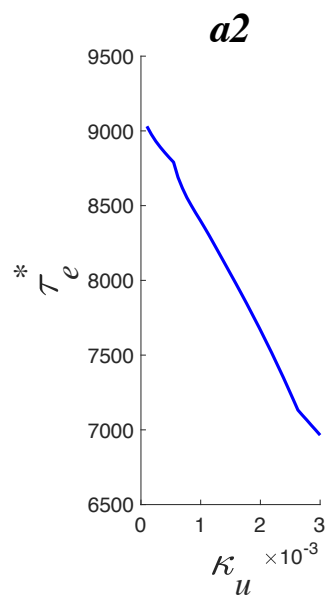

b2

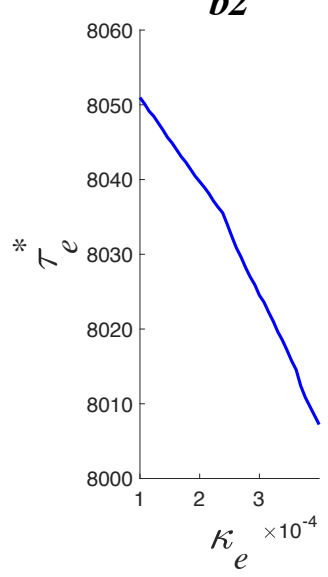

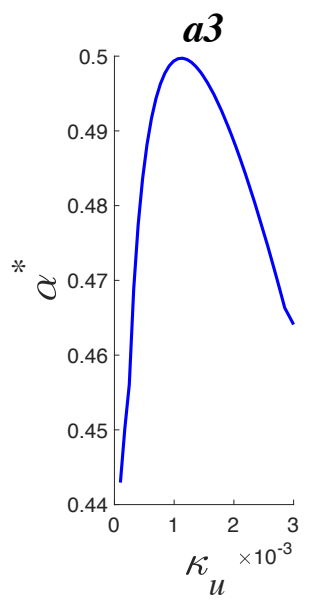

b3

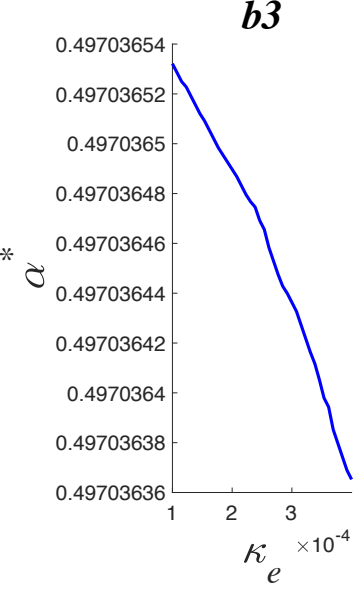

This figure plots values of $\left(\tau_{u}^{*}, \tau_{e}^{*}, \alpha^{*}\right)$ for the second equilibrium as functions of $\kappa_{u}$ in the top panels and $\kappa_{e}$ in the bottom panels. The cost function is $C\left(\tau_{u, i}, \tau_{e, i}\right)=\kappa_{u} \tau_{u, i}+\kappa_{e} \tau_{e, i}$, where $\kappa_{u}$ and $\kappa_{e}$ are positive constants. The parameters are set as follows: $\tau_{v}=25, \tau_{\varepsilon}=1$ and $\gamma=2$.

We can see this non-monotonicity in Panel a of Figure 5, where we use the same parameters as in Figure 1. Intuitively, when $\tau_{u}=0$ (low $\tau_{u}$ ), there is no noise $\tilde{u}$ in the price. As $\tau_{u}$ increases, the noise $\tilde{u}$ gets incorporated into the price and the incentives to tame sentiment increase $\left(\phi\left(\tau_{u}\right)\right.$ is increasing). In contrast, for high values of $\tau_{u}$, the price signal is already very informative and a higher $\tau_{u}$ will not provide much additional information $\left(\phi\left(\tau_{u}\right)\right.$ is decreasing). Consequently, the marginal benefit $\phi\left(\tau_{u}\right)$ has a hump-shape and there are multiple equilibria.

In the following proposition, we formally analyze the potential presence of multiple equilibria for a general cost function. Since we fix $\tau_{e, i}$ and only consider the choice of $\tau_{u, i}$, we denote the 

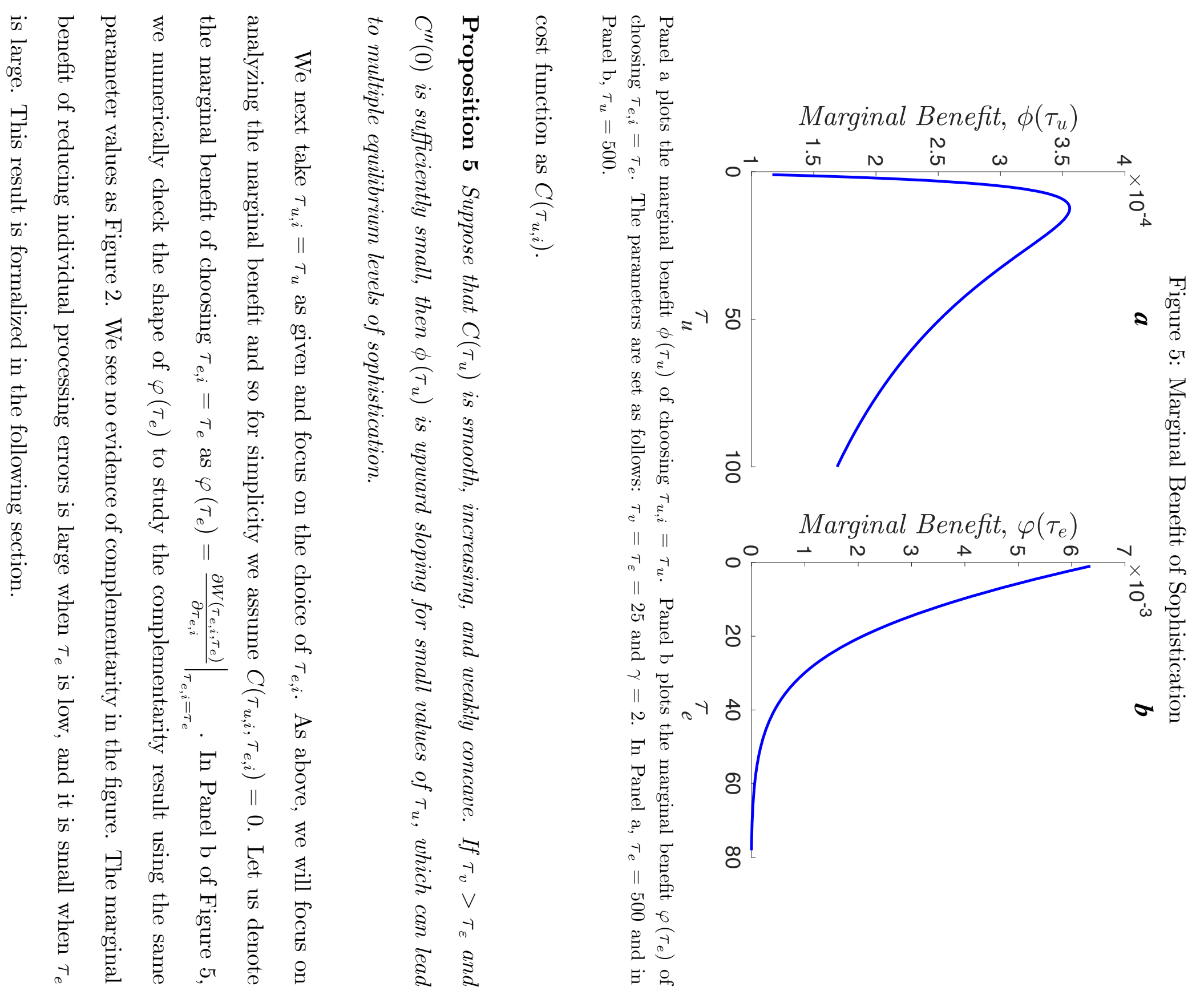


\subsection{Uncorrelated Receiver Noise}

This section solves the model with no common sentiment errors $\tilde{u}$. To this end, we assume that $\tau_{u, i}=\tau_{u} \rightarrow \infty$. Corollary 1 shows that in a symmetric equilibrium with $\tau_{e, i}=\tau_{e}$, price coefficients are given by $a_{v}=\frac{\tau_{e}+\tau_{\varepsilon}}{\tau_{e}+\tau_{v}+\tau_{\varepsilon}}$ and $a_{u}=0$, implying that $\alpha=0$. In this case, returns still exhibit momentum:

$$
m=\frac{\operatorname{Cov}(\tilde{v}-\tilde{p}, \tilde{p})}{\operatorname{Var}(\tilde{p})}=\frac{\tau_{v}}{\tau_{\varepsilon}+\tau_{e}}>0 .
$$

Thus, the results on return predictiveness of the model are robust to turning off the common sentiment error term $\tilde{u}$. Trading volume is given by

$$
\text { Volume }=\frac{1}{\gamma} \sqrt{\frac{2}{\pi}\left(\tau_{\varepsilon}+\tau_{e}\right)}
$$

and it is increasing in sophistication $\tau_{e}$. The following proposition formally analyzes the existence of a unique equilibrium in sophistication levels when the cost function is $C\left(\tau_{e, i}\right)=\kappa_{e} \tau_{e, i}$.

Proposition 6 Suppose that $\tau_{u} \rightarrow \infty$ and $C\left(\tau_{e, i}\right)=\kappa_{e} \tau_{e, i}$. If $\kappa_{e}$ is sufficiently small, then there is a unique symmetric equilibrium with $\tau_{e}^{*}>0$. Otherwise, there is a unique symmetric equilibrium with $\tau_{e}^{*}=0$.

This proposition suggests that complementarity and multiplicity are driven by the choice of $\tau_{u}$. When we get rid of the common error term $\tilde{u}$ in the receiver noise, the complementarity result disappears.

\section{Extension with Noisy Supply}

In this section, we extend the setup in Section 3.1 with exogenous noisy trading. Specifically, the risky asset has a noisy supply $\tilde{q} \sim N\left(0, \tau_{q}^{-1}\right)$, which is independent of all the other random variables. All the other features of the setup in Section 3.1 remain unchanged. The market- 
clearing condition in the financial market now becomes

$$
\int_{0}^{1} D\left(\tilde{p} ; \tilde{s}_{i}, \tilde{s}_{p, i}\right) d i=\tilde{q} \text { almost surely. }
$$

With noisy supply, the price of the risky asset is given by

$$
\tilde{p}=a_{v} \tilde{v}+a_{u} \tilde{u}+a_{q} \tilde{q}
$$

where $a$ 's are endogenous coefficients. Hence, the price signal can be written as

$$
\tilde{s}_{p, i}=\tilde{v}+\alpha \tilde{u}+\theta \tilde{q}+\frac{1}{\sqrt{\tau_{u, i}}} \tilde{u}+\frac{1}{\sqrt{\tau_{e, i}}} \tilde{e}_{i}
$$

where $\alpha \equiv \frac{a_{u}}{a_{v}}$ and $\theta \equiv \frac{a_{q}}{a_{v}}$. The investor's beliefs in this case are given by

$$
\begin{aligned}
E\left(\tilde{v} \mid \tilde{s}_{i}, \tilde{s}_{p, i}\right) & =\left(\tau_{\varepsilon} \tilde{s}_{i}+\frac{\tau_{e, i} \tau_{u, i} \tau_{q}}{\tau_{u, i} \tau_{q}+\tau_{e, i} \tau_{q}\left(\alpha \sqrt{\tau_{u, i}}+1\right)^{2}+\tau_{e, i} \tau_{u, i} \theta^{2}} s_{p, i}\right) V\left(\tilde{v} \mid \tilde{s}_{i}, \tilde{s}_{p, i}\right), \\
V\left(\tilde{v} \mid \tilde{s}_{i}, \tilde{s}_{p, i}\right) & =\frac{\tau_{u, i} \tau_{q}+\tau_{e, i} \tau_{q}\left(\alpha \sqrt{\tau_{u, i}}+1\right)^{2}+\tau_{e, i} \tau_{u, i} \theta^{2}}{\tau_{e, i} \tau_{u, i} \tau_{q}+\left(\tau_{u, i} \tau_{q}+\tau_{e, i} \tau_{q}\left(\alpha \sqrt{\tau_{u, i}}+1\right)^{2}+\tau_{e, i} \tau_{u, i} \theta^{2}\right)\left(\tau_{v}+\tau_{\varepsilon}\right)} .
\end{aligned}
$$

The following proposition shows the financial market equilibrium and return predictiveness in this setup with noisy supply.

Proposition 7 Suppose that investors have the same sophistication level (i.e., $\tau_{u, i}=\tau_{u}$ and $\left.\tau_{e, i}=\tau_{e}, i \in[0,1]\right)$ in the economy with noisy supply. Then:

(a) There exists a unique linear equilibrium price function with coefficients:

$$
\begin{aligned}
& a_{v}=\frac{\tau_{e} \tau_{u} \tau_{q}+\tau_{\varepsilon}\left(\tau_{u} \tau_{q}+\tau_{e} \tau_{q}\left(\alpha \sqrt{\tau_{u}}+1\right)^{2}+\tau_{e} \tau_{u} \theta^{2}\right)}{\tau_{e} \tau_{u} \tau_{q}+\left(\tau_{u} \tau_{q}+\tau_{e} \tau_{q}\left(\alpha \sqrt{\tau_{u}}+1\right)^{2}+\tau_{e} \tau_{u} \theta^{2}\right)\left(\tau_{v}+\tau_{\varepsilon}\right)} \\
& a_{u}= \frac{\tau_{e} \sqrt{\tau_{u}} \tau_{q}\left(\alpha \sqrt{\tau_{u}}+1\right)}{\tau_{e} \tau_{u} \tau_{q}+\left(\tau_{u} \tau_{q}+\tau_{e} \tau_{q}\left(\alpha \sqrt{\tau_{u}}+1\right)^{2}+\tau_{e} \tau_{u} \theta^{2}\right)\left(\tau_{v}+\tau_{\varepsilon}\right)} \\
& a_{q}=-\frac{\gamma\left(\tau_{u} \tau_{q}+\tau_{e} \tau_{q}\left(\alpha \sqrt{\tau_{u}}+1\right)^{2}+\tau_{e} \tau_{u} \theta^{2}\right)-\tau_{e} \tau_{u} \tau_{q} \theta}{\tau_{e} \tau_{u} \tau_{q}+\left(\tau_{u} \tau_{q}+\tau_{e} \tau_{q}\left(\alpha \sqrt{\tau_{u}}+1\right)^{2}+\tau_{e} \tau_{u} \theta^{2}\right)\left(\tau_{v}+\tau_{\varepsilon}\right)}
\end{aligned}
$$


where

$$
\theta=-\frac{\gamma}{\tau_{\varepsilon}}
$$

and $\alpha$ is determined by the unique real root of the following cubic equation:

$$
\alpha^{3}+\frac{2}{\sqrt{\tau_{u}}} \alpha^{2}+\left(\frac{\gamma^{2}}{\tau_{q} \tau_{\varepsilon}^{2}}+\frac{1}{\tau_{e}}+\frac{1}{\tau_{u}}\right) \alpha-\frac{1}{\sqrt{\tau_{u}} \tau_{\varepsilon}}=0 .
$$

(b) There exists a threshold $\hat{\tau}_{q}$ such that returns exhibit momentum $m>0$ for any $\tau_{q}>\hat{\tau}_{q}$.

Figures 6 report the results for the two main results of the paper, i.e., return predictiveness $m$ and complementarity in the sentiment loading $\tau_{u, i}$. We use the same parameter values as in Figure 1 and set $\tau_{q}=0.1$ in the top panels and $\tau_{q}=5$ in the bottom panels. We see that the complementarity result in the sophistication choice prevails in both panels.

However, whether the momentum result arises depends on the size of noise trading. We can see that the sign of $m$ is negative when $\tau_{q}$ is low, but it is positive when $\tau_{q}$ is high. Intuitively, the sign of return predictiveness is determined by two counteracting forces. First, as in the traditional noisy-REE setting, exogenous noise trading delivers return reversals (see Banerjee et al., 2009; Vives, 2008): exogenous noise demand pushes the current price too high and exogenous noisy supply depresses the current price too low. Second, in our setting, investors interpret price costly, which causes the price to underreact to information, leading to return momentum. When the size of noise trading is high (low $\tau_{q}$ ), the first effect dominates. In contrast, when the size of noise trading is low (high $\tau_{q}$ ), the second effect dominates.

\section{Comparison to Other Related Models}

In this section, we examine other models that are conceptually related to our story. We consider four alternative theories: (a) information acquisition about noisy supply, (b) dismissive traders, (c) cursed traders, and (d) operation risk. We will show that supply information and operation risk deliver return reversal and that dismissiveness and cursedness can deliver price momentum. 


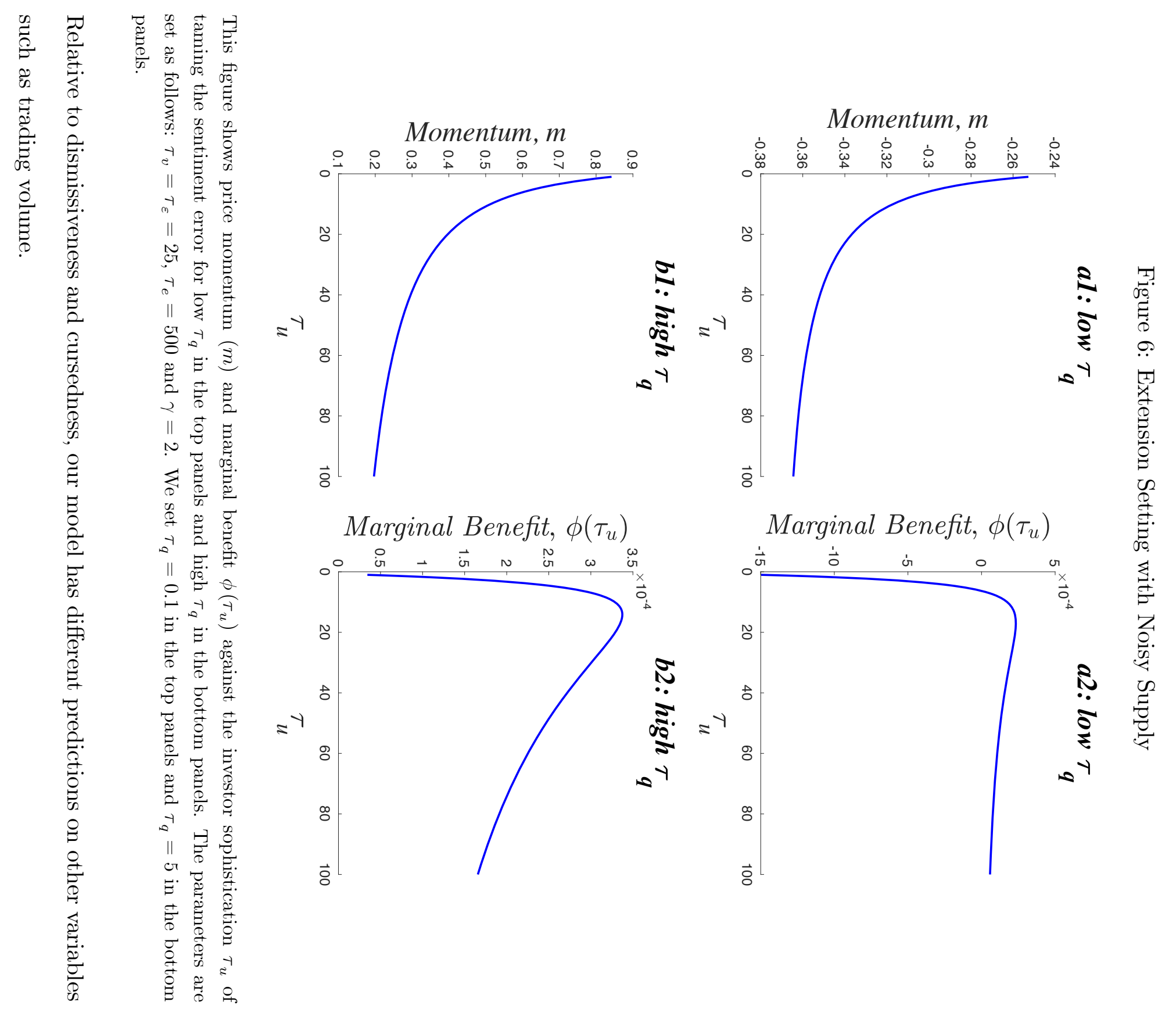




\subsection{Information Acquisition about Noisy Supply}

Information acquisition about noisy supply has been analyzed by Ganguli and Yang (2009), Manzano and Vives (2011), and Marmora and Rytchkov (2018), among others. We base our analysis on Ganguli and Yang (2009) and modify our setup in Section 3.1 in two aspects. First, we shut down the sophistication level choice in our model by setting both $\tau_{u} \rightarrow \infty$ and $\tau_{e} \rightarrow \infty$, which implies that investors are able to observe the best price signal $\tilde{s}_{p}$. Second, we assume that at date 1 , investor $i$ is endowed with $q_{i}$ units of the risky asset, where $\tilde{q}_{i}=\tilde{q}+\tilde{\eta}_{i}$ with $\tilde{q} \sim N\left(0, \tau_{q}^{-1}\right)$ and $\tilde{\eta}_{i} \sim N\left(0, \tau_{\eta}^{-1}\right)$, which implies that the aggregate supply is

$$
\int_{0}^{1} \tilde{q}_{i} d i=\tilde{q}
$$

With these two modifications, the price of the risky asset is given by

$$
\tilde{p}=a_{v} \tilde{v}-a_{q} \tilde{q}
$$

In this supply-information model, there can exist multiple linear equilibria at the trading stage. In each of these equilibria, returns exhibit reversal (i.e., $m<0$ ), which contrasts to our setting of costly interpretation of prices.

Proposition 8 In the economy with supply information, if $\gamma^{2}>4 \tau_{\varepsilon} \tau_{\eta}$, then there exist two equilibria with price coefficients given by

$$
\begin{aligned}
& a_{v}=\left(\tau_{v}+\tau_{\varepsilon}+\tau_{p}\right)^{-1}\left(\tau_{\varepsilon}+\tau_{p}\right), \\
& a_{q}=\left(\tau_{v}+\tau_{\varepsilon}+\tau_{p}\right)^{-1}\left(\tau_{q}+\gamma\right),
\end{aligned}
$$

with $\tau_{p}=\beta^{2}\left(\tau_{q}+\tau_{\eta}\right)$ and $\beta=a_{v} / a_{q}$ takes two values

$$
\begin{aligned}
& \beta_{S U B}=\left(2 \tau_{\eta}\right)^{-1}\left(\gamma-\sqrt{\gamma^{2}-4 \tau_{\varepsilon} \tau_{\eta}}\right), \\
& \beta_{C O M}=\left(2 \tau_{\eta}\right)^{-1}\left(\gamma+\sqrt{\gamma^{2}-4 \tau_{\varepsilon} \tau_{\eta}}\right) .
\end{aligned}
$$


Under SUB-equilibrium, information acquisition is a strategic substitute. Under COM-equilibrium, information acquisition is a complement. Returns exhibit reversal under both equilibria:

$$
m=\frac{\operatorname{Cov}(\tilde{v}-\tilde{p}, \tilde{p})}{\operatorname{Var}(\tilde{p})}<0 .
$$

\subsection{Dismissiveness}

We incorporate dismissiveness into our model based on Banerjee (2011). We make two modifications to our setup with noise trading in Section 6. First, we shut down the sophistication level choice in our model by setting both $\tau_{u} \rightarrow \infty$ and $\tau_{e} \rightarrow \infty$, which implies that investors are able to observe the best price signal $\tilde{s}_{p}$. Second, investor $i$ 's beliefs about the private signal of investor $j$ are given by

$$
\tilde{s}_{i, j}=\rho \tilde{v}+\sqrt{\left(1-\rho^{2}\right)} \tilde{\phi}+\tilde{\varepsilon}_{j} \text {, where } \tilde{\varepsilon}_{j} \sim N\left(0, \tau_{\varepsilon}^{-1}\right), \tilde{\phi} \sim N\left(0, \tau_{v}^{-1}\right),
$$

$\rho \in[0,1]$ and $\tilde{\phi}$ is independent of $\tilde{v}$. The parameter $\rho$ measures the degree of dismissiveness by investors. When $\rho<1$, each investor believes that the private signal of all other investors includes an additional random variable $\tilde{\phi}$. When $\rho=1$, then we have a standard REE model. With these modifications, under the beliefs of the investors, the price of the risky asset is given by

$$
\tilde{p}=a_{v} \rho \tilde{v}+a_{v} \sqrt{\left(1-\rho^{2}\right)} \tilde{\phi}+a_{q} \tilde{q} .
$$

Dismissiveness can predict either momentum or reversal, depending on the value of investors' risk aversion: When the risk aversion is sufficiently small, returns exhibit momentum, and when the risk aversion is sufficiently large, returns exhibit reversal. This is intuitive because the risk aversion scales how much noise there is in prices, and so how much noise-driven reversal there is. Nonetheless, dismissiveness delivers different volume predictions from our model. Specifically, trading volume increases with dismissiveness and hence decreases with sophistication (to the extent that dismissiveness is inversely related to sophistication). By contrast, in our setting, 
trading volume either increases with sophistication or is hump-shaped in sophistication.

Proposition 9 In a setting with dismissive investors, there is a unique linear equilibrium price function with coefficients:

$$
\begin{aligned}
& a_{v}=\frac{\left(\tau_{v} \tau_{\varepsilon} \alpha^{2}-\tau_{q} \tau_{\varepsilon} \rho^{2}+\tau_{q} \tau_{v} \rho+\tau_{q} \tau_{\varepsilon}\right)}{\left(\alpha^{2} \tau_{v}^{2}+\tau_{\varepsilon} \alpha^{2} \tau_{v}-\tau_{q} \tau_{\varepsilon} \rho^{2}+\tau_{q} \tau_{v}+\tau_{q} \tau_{\varepsilon}\right)} \\
& a_{q}=-\frac{\left(\gamma \tau_{v} \alpha^{2}-\tau_{q} \tau_{v} \alpha \rho-\gamma \tau_{q} \rho^{2}+\gamma \tau_{q}\right)}{\left(\alpha^{2} \tau_{v}^{2}+\tau_{\varepsilon} \alpha^{2} \tau_{v}-\tau_{q} \tau_{\varepsilon} \rho^{2}+\tau_{q} \tau_{v}+\tau_{q} \tau_{\varepsilon}\right)}
\end{aligned}
$$

where $\alpha \equiv \frac{a_{q}}{a_{v}}=-\gamma / \tau_{\varepsilon}$. For sufficiently small $\gamma$, returns exhibit momentum, and for sufficiently large $\gamma$, returns exhibit reversal. Trading volume is increasing in dismissiveness.

\subsection{Cursed Traders}

We analyze cursed traders based on Eyster et al. (2019). We modify our setup in Section 3.1 in four aspects. First, we shut down the sophistication level choice in our model by setting both $\tau_{u} \rightarrow \infty$ and $\tau_{e} \rightarrow \infty$, which implies that investors are able to observe the best price signal $\tilde{s}_{p}$. Second, we assume there is a finite number of traders $N$. Third, the risky asset pays date-2 value $\tilde{v}+\tilde{\varsigma}$, where $\tilde{\varsigma} \sim N\left(0, \tau_{\varsigma}^{-1}\right)$ is an unlearnable risk, so the private signal is only about the payoff component $\tilde{v}$. Fourth, investors' behavior lies between rationality and full cursedness. Traders maximize the following utility

$$
E U=E\left[-e^{-\gamma D_{i}(\tilde{v}+\tilde{\varsigma}-\tilde{p})} \mid \tilde{s}_{i}, \tilde{p}\right]^{1-\chi} E\left[-e^{-\gamma D_{i}(\tilde{v}+\tilde{\varsigma}-\tilde{p})} \mid \tilde{s}_{i}\right]^{\chi}
$$

where parameter $\chi$ captures the extent of cursedness. With these modifications, the price of the risky asset is given by

$$
\tilde{p}=a_{v} \sum_{i=1}^{N} \tilde{s}_{i} .
$$

This model is able to generate positive momentum as in our model. Intuitively, cursed traders $(\chi>0)$ disregard information from the price, which leads to price underreaction. The mechanism is similar to our model. As explained in Section 2, our framework complements Eyster et al. 
(2019) in three important ways. First, Eyster et al. (2019) predict that trading volume always increases with cursedness and hence decreases with sophistication. By contrast, in our setting, volume is either increasing or hump-shaped in sophistication. Intuitively, since cursed volume arises from investors neglecting price information, the more cursed and less sophisticated are investors, the higher is volume. In our setting, if investors are less exposed to the sentiment error, their posterior beliefs put relative higher weights on individual processing errors, generating more disagreement and higher volume. This difference in sophistication-volume patterns can be taken to the data to differentiate our model from cursedness (for instance, one can use the fraction of institutional trading to proxy for the sophistication degree of a market).

Second and more importantly, our setting has an extra stage to determine the equilibrium level of investor sophistication, which generates novel theory results such as strategic complementarity in sophistication acquisition. Third, our analysis incorporates a common error in interpreting prices, which generates a form of endogenous noise trading. ${ }^{8}$

Proposition 10 In the economy with cursed traders, the price coefficient is given by

$$
a_{v}=\frac{\tau_{\varepsilon}\left([N-\chi(N-1)] \tau_{v}+N \tau_{\varepsilon}\right)}{N\left(\tau_{v}+\tau_{\varepsilon}\right)\left(\tau_{v}+N \tau_{\varepsilon}\right)}
$$

This model with cursed traders generates momentum:

$$
m=\frac{\operatorname{Cov}(\tilde{v}-\tilde{p}, \tilde{p})}{\operatorname{Var}(\tilde{p})}=a_{v} \frac{\chi(N-1)\left(\tau_{v}+N \tau_{\varepsilon}\right)}{\left(\tau_{v}+\tau_{\varepsilon}\right)\left(\tau_{v}+N \tau_{\varepsilon}\right)}>0
$$

Trading volume is increasing in cursedness $\chi$.

\subsection{Operation Risk}

We incorporate operation risk into our model based on Basak and Buffa (2019). We modify our setup with noise trading in Section 6 in two aspects. First, we shut down the sophistication level

\footnotetext{
${ }^{8}$ We have also solved our Setup in Section 3.1 with a finite number of investors $N$ to make it more comparable to Eyster et al. (2019). The main implications of the model are robust to a finite number of traders.
} 
choice in our model by setting $\tau_{u} \rightarrow \infty$ and $\tau_{e} \rightarrow \infty$, which implies that investors are able to observe the best price signal $\tilde{s}_{p}$. Second, the asset demand contains operational errors, hence the asset demand is given by

$$
D_{o r, i}=D_{i}+\tilde{x}+\tilde{\eta}_{i}, \text { where } \tilde{x} \sim N\left(0, \tau_{x}^{-1}\right) \text { and } \tilde{\eta}_{i} \sim N\left(0, \tau_{\eta}^{-1}\right)
$$

$D_{i}$ is the optimal investment strategy and $\tilde{x}+\tilde{\eta}_{i}$ is the operational error. The operational error $\tilde{x}$ is common to all investors, while operational errors $\tilde{\eta}_{i}$ are identically and independently distributed.

With these modifications, the price of the risky asset is given by

$$
\tilde{p}=a_{v} \tilde{v}+a_{x} \tilde{x}+a_{q} \tilde{q}
$$

Hence, the price signal can be written as

$$
\tilde{s}_{p, i}=\tilde{v}+\alpha \tilde{x}+\theta \tilde{q}
$$

where $\alpha \equiv \frac{a_{x}}{a_{v}}$ and $\theta \equiv \frac{a_{q}}{a_{v}}$. Solving for the optimal asset demand is not straightforward because final wealth is no longer normally distributed and we cannot use mean-variance results. Final wealth is given by

$$
W_{i}=D_{i}(\tilde{v}-\tilde{p})+\tilde{x}(\tilde{v}-\tilde{p})+\tilde{\eta}_{i}(\tilde{v}-\tilde{p})
$$

and we need to take expectations:

$$
E U=E\left[-\exp \left(-\gamma W_{i}\right) \mid \tilde{s}_{i}, \tilde{s}_{p, i}\right]
$$

When there is no common operational risk $\tilde{x}$, the optimal asset demand $D_{i}$ turns out to be the exact same as the standard CARA-Normal framework:

$$
D\left(\tilde{p} ; \tilde{s}_{i}, \tilde{s}_{p, i}\right)=\frac{E\left(\tilde{v} \mid \tilde{s}_{i}, \tilde{s}_{p, i}\right)-\tilde{p}}{\gamma \operatorname{Var}\left(\tilde{v} \mid \tilde{s}_{i}, \tilde{s}_{p, i}\right)} .
$$


With common operational risk $\tilde{x}$, the optimal demand is given by

$$
D\left(\tilde{p} ; \tilde{s}_{i}, \tilde{s}_{p, i}\right)=\frac{E\left(\tilde{v} \mid \tilde{s}_{i}, \tilde{s}_{p, i}\right)-\tilde{p}}{\gamma \operatorname{Var}\left(\tilde{v} \mid \tilde{s}_{i}, \tilde{s}_{p, i}\right)}-E\left(\tilde{x} \mid \tilde{s}_{i}, \tilde{s}_{p, i}\right)
$$

Hence, the total demand is

$$
D_{o r, i}=\frac{E\left(\tilde{v} \mid \tilde{s}_{i}, \tilde{s}_{p, i}\right)-\tilde{p}}{\gamma \operatorname{Var}\left(\tilde{v} \mid \tilde{s}_{i}, \tilde{s}_{p, i}\right)}+\left[\tilde{x}-E\left(\tilde{x} \mid \tilde{s}_{i}, \tilde{s}_{p, i}\right)\right]+\tilde{\eta}_{i} .
$$

Intuitively, since investors know that they will get a common shock $\tilde{x}$ to their ideal demand, then they correct demand by their best forecast of $\tilde{x}$. They are able to estimate $\tilde{x}$ with both signals $\tilde{s}_{i}$ and $\tilde{s}_{p, i}$

Proposition 11 The coefficients in the linear equilibrium price function are given by:

$$
\begin{aligned}
& a_{v}=\frac{\left[\tau_{q} \tau_{x}+\tau_{\varepsilon}\left(\alpha^{2} \tau_{q}+\theta^{2} \tau_{x}\right)\right]\left[\tau_{q} \tau_{x}+\left(\tau_{v}+\tau_{\varepsilon}\right)\left(\alpha^{2} \tau_{q}+\theta^{2} \tau_{x}\right)\right]-\alpha \gamma \tau_{q} \tau_{v}\left(\alpha^{2} \tau_{q}+\theta^{2} \tau\right)}{\left[\tau_{q} \tau_{x}+\left(\tau_{v}+\tau_{\varepsilon}\right)\left(\alpha^{2} \tau_{q}+\theta^{2} \tau_{x}\right)\right]^{2}} \\
& a_{x}=\frac{\left[\alpha \tau_{q} \tau_{x}+\gamma\left(\alpha^{2} \tau_{q}+\theta^{2} \tau_{x}\right)\right]\left[\tau_{q} \tau_{x}+\left(\tau_{v}+\tau_{\varepsilon}\right)\left(\alpha^{2} \tau_{q}+\theta^{2} \tau_{x}\right)\right]-\alpha^{2} \gamma \tau_{q}\left(\tau_{v}+\tau_{\varepsilon}\right)\left(\alpha^{2} \tau_{q}+\theta^{2} \tau_{x}\right)}{\left[\tau_{q} \tau_{x}+\left(\tau_{v}+\tau_{\varepsilon}\right)\left(\alpha^{2} \tau_{q}+\theta^{2} \tau_{x}\right)\right]^{2}} \\
& a_{u}=-a_{q}
\end{aligned}
$$

where $\theta=\frac{a_{q}}{a_{v}}, \alpha=\frac{a_{x}}{a_{v}}, \theta=-\alpha$, and $\alpha$ is a real root to the following cubic equation:

$$
\left(\tau_{q} \tau_{\varepsilon}^{2}+\tau_{x} \tau_{\varepsilon}^{2}+\tau_{q} \tau_{v} \tau_{\varepsilon}+\tau_{x} \tau_{v} \tau_{\varepsilon}\right) \alpha^{3}+\left(\gamma \tau_{q} \tau_{v}+\gamma \tau_{x} \tau_{v}+\gamma \tau_{x} \tau_{\varepsilon}\right) \alpha^{2}+\tau_{q} \tau_{x} \tau_{\varepsilon} \alpha+\gamma \tau_{q} \tau_{x}=0
$$

The common error $\tilde{x}$ behaves in a very similar way as noisy supply, with the only difference that agents can learn about $\tilde{x}$ and as a consequence, investors try to correct for the demand shock $\tilde{x}$ in their ideal demand $D_{i}$. Figure 7 uses the same parameter values as Figure 6 with high $\tau_{q}$, where investors are not able to observe the ideal price signal and there is noisy supply. We assume that the precision $\tau_{x}$ of the common error $\tilde{x}$ is the same as the one assumed for the sentiment term $\tilde{u}$. Figure 7 shows that the model with operation risk fails to generate momentum for those values. As mentioned above, common operation risks behave in a similar way as a noisy 
supply, and thus are not able to generate momentum. We have tried a variety of parameter values and find that the results are robust even for very large values of $\tau_{q}$. Panel b shows that momentum under operation risk does not depend on the precision $\tau_{\eta}$ of idiosyncratic operational errors. Intuitively, these idiosyncratic errors disappear when aggregating demand and they have no effect on asset prices.

Figure 7: Return Predictiveness in Settings of Operation Risk
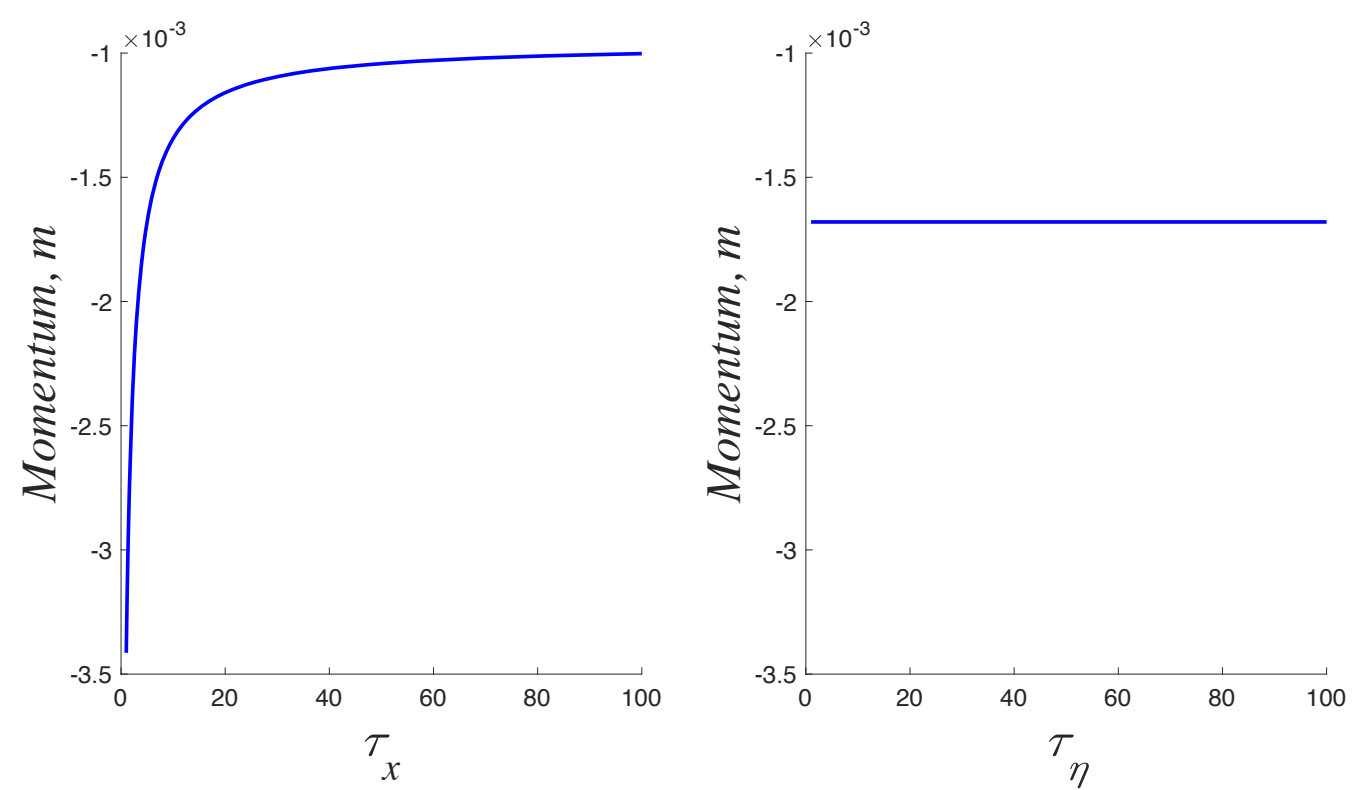

This figure shows price momentum $(m)$ against the precision of the common operational risk $\tilde{x}$ and the precision of the idiosyncratic operational risk $\tilde{\eta}_{i}$. The parameters are set as follows: $\tau_{v}=\tau_{\varepsilon}=25, \tau_{q}=5$ and $\gamma=2$. Panel a sets $\tau_{\eta}=5$ and Panel b sets $\tau_{x}=5$.

Corollary 2 In the setting with operation risk, if $\tau_{x} \rightarrow \infty$, then there is a unique linear equilibrium price function with coefficients:

$$
\begin{aligned}
a_{v} & =\frac{\tau_{\varepsilon} \theta^{2}+\tau_{q}}{\tau_{q}+\theta^{2} \tau_{v}+\theta^{2} \tau_{\varepsilon}} \\
a_{q} & =\frac{-\gamma \theta^{2}+\tau_{q} \theta}{\tau_{q}+\theta^{2} \tau_{v}+\theta^{2} \tau_{\varepsilon}}
\end{aligned}
$$

where $\theta=\frac{a_{q}}{a_{v}}=-\gamma / \tau_{\varepsilon}$. The asset price is isomorphic to a standard REE equilibrium and returns exhibit reversal (i.e., $m<0$ ).

In the limiting case without common error, since all operational errors are identically and 
independently distributed, then we end up with a standard REE equilibrium because all operational errors cancel with each other when aggregated. Return volatility and momentum will be the same as in a standard REE equilibrium, but volume will be higher because of the operational errors. In particular, this model of operation risk is not able to generate price momentum.

\section{Conclusion}

We develop a model to capture the notion that investors cannot costlessly process price data in financial markets. Although investors actively infer information from the price, their information processing is noisy. The more sophisticated are investors, the smaller is this processing noise. After reading price data and form their beliefs, investors hold optimal trading positions according to their own beliefs (and so they are only boundedly rational in extracting information from the price). We find that imperfect price interpretation can inject noise into the price system, which serves as a form of endogenous noise trading in our setting. Our model predicts price momentum because investors fail to make full use of the information in asset prices. Compared to the standard REE, our model generate excessive return volatility and excessive trading volume. There can exist strategic complementarity in acquiring sophistication of taming sentiment, leading to the possibility of multiple equilibria. Our theory qualitatively differs from many

other conceptually related models such as supply information, dismissiveness, cursedness, and operation risk. 


\section{References}

[1] Addoum, J. M. and J. R. Murfin (2017), "Hidden in Plain Sight: Equity Price Discovery with Informed Private Debt," Working Paper.

[2] Angeletos, G-M. and K. A. Sastry (2019), "Inattentive Economies," Working Paper.

[3] Banerjee, S. (2011), "Learning from Prices and the Dispersion in Beliefs," Review of Financial Studies 24, 3025-3068.

[4] Banerjee, S., J. Davis, and N. Gondhi (2019), "Choosing to Disagree in Financial Markets," Working paper.

[5] Banerjee, S., R. Kaniel, and I. Kremer (2009), "Price Drift as an Outcome of Differences in Higher Order Beliefs", Review of Financial Studies 22, 3707-3734.

[6] Barber, B. and T. Odean (2000), "Trading Is Hazardous to Your Wealth: The Common Stock Performance of Individual Investors," Journal of Finance 55, 773-806.

[7] Basak, S. and A. M. Buffa (2019), "A Theory of Model Sophistication and Operational Risk," Available at SSRN: https://ssrn.com/abstract=2737178.

[8] Esponda, I. and D. Pouzo (2016), "Berk-Nash Equilibrium: A Framework for Modeling Agents with Misspecified Models," Econometrica 84, 1093-1130.

[9] Eyster, E. and M. Rabin (2005), "Cursed Equilibrium," Econometrica 73, 1623-1672.

[10] Eyster, E., M. Rabin, and D. Vayanos (2019), "Financial Markets where Traders Neglect the Informational Content of Prices," Journal of Finance 74, 371-399.

[11] Gargano, A. and A. G. Rossi (2018), "Does it Pay to Pay Attention?", Review of Financial Studies, Forthcoming.

[12] Grossman, S. and J. Stiglitz (1980), "On the Impossibility of Informationally Efficient Markets," American Economic Review 70, 393-408.

[13] Guesnerie, R. (1992), "An Exploration of the Eductive Justifications of the RationalExpectations Hypothesis," American Economic Review 82, 1254-1278.

[14] Hellwig, M. (1980), "On the Aggregation of Information in Competitive Markets," Journal of Economic Theory 22, 477-498.

[15] Hong, H. and J. Stein (1999), "A Unified Theory of Underreaction, Momentum Trading and Overreaction in Asset Markets," Journal of Finance 54, 2143-2184.

[16] Jegadeesh, N. and S. Titman (1993), "Returns to Buying Winners and Selling Losers: Implications for Stock Market Efficiency," Journal of Finance 48, 65-91.

[17] Kovalenkov, A. and X. Vives (2014), "Competitive Rational Expectations Equilibria without Apology," Journal of Economic Theory 149, 211-235. 
[18] LeRoy, S. and R. Porter (1981), "The Present-value Relation: Tests Based on Implied Variance Bounds," Econometrica 49, 97-113.

[19] Manzano, C. and X. Vives (2011), "Public and Private Learning from Prices, Strategic Substitutability and Complementarity, and Equilibrium Multiplicity," Journal of Mathematical Economics 47, 346-369.

[20] Marmora, P. and O. Rytchkov (2018), "Learning about Noise," Journal of Banking and Finance 89, 209-224

[21] Moskowitz, T., Y. H. Ooi, and L. H. Pedersen (2012), "Time Series Momentum," Journal of Financial Economics 104, 228-250.

[22] Odean, T. (1999), "Do Investors Trade Too Much?", American Economic Review 89, 12791298.

[23] Peress, J. (2010), "The Tradeoff between Risk Sharing and Information Production in Financial Markets," Journal of Economic Theory 145, 124-155.

[24] Shiller, R. (1981), "Do Stock Prices Move Too Much to Be Justified by Subsequent Changes in Dividends?", American Economic Review 71, 421-436.

[25] Verrecchia, R. E. (1982), "Information Acquisition in a Noisy Rational Expectations Economy," Econometrica 50, 1415-1430.

[26] Vives, X. (2008), Information and Learning in Markets: The Impact of Market Microstructure. Princeton University Press, Princeton and Oxford. 


\section{Appendices (For Online Publication)}

\section{A Proof of Proposition 1}

Using Bayes' rule, we can compute investors' beliefs given by equations (11) and (12). In a symmetric equilibrium with $\tau_{u, i}=\tau_{u}$ and $\tau_{e, i}=\tau_{e}$, all investors have the same conditional variance $\operatorname{Var}\left(\tilde{v} \mid \tilde{s}_{i}, \tilde{s}_{p, i}\right)$. Thus, inserting these two expressions into the demand (5), we can compute the expression of $D\left(\tilde{p} ; \tilde{s}_{i}, \tilde{s}_{p, i}\right)$, which is in turn inserted into (6) to compute the equilibrium price as a function of $\tilde{v}$ and $\tilde{u}$. Comparing coefficients with the conjectured price function (8), we can form a system of equations to determine the two unknown price coefficients $a_{v}$ and $a_{u}$ in Proposition 1. Inserting the expressions of $a$ 's into $\alpha \equiv \frac{a_{u}}{a_{v}}$ and simplifying yields the cubic (13) that determines the value of $\alpha$. Denote the left-hand side of $(13)$ by $f(\alpha)$. That is,

$$
f(\alpha) \equiv \tau_{e} \tau_{u} \tau_{\varepsilon} \alpha^{3}+2 \tau_{e} \sqrt{\tau_{u}} \tau_{\varepsilon} \alpha^{2}+\left(\tau_{e} \tau_{\varepsilon}+\tau_{u} \tau_{\varepsilon}\right) \alpha-\tau_{e} \sqrt{\tau_{u}}
$$

We can compute $f(0)=-\tau_{e} \sqrt{\tau_{u}}<0$ and $f\left(\frac{\tau_{e} \sqrt{\tau_{u}}}{\tau_{e} \tau_{\varepsilon}+\tau_{u} \tau_{\varepsilon}}\right)>0$, and thus by the Intermediate Value Theorem, there exists a solution $\alpha \in\left(0, \frac{\tau_{e} \sqrt{\tau_{u}}}{\tau_{e} \tau_{\varepsilon}+\tau_{u} \tau_{\varepsilon}}\right)$ such that $f(\alpha)=0$. This result establishes the existence of a financial market equilibrium. The discriminant of the cubic (13) is negative. Thus, there exists a unique real root, which establishes the uniqueness of a financial market equilibrium. QED.

\section{B Proof of Corollary 1}

Given $\left(\tau_{v}, \tau_{\varepsilon}\right) \in \mathbb{R}_{++}^{2}$, if we take the following limits of the price coefficients $a_{v}$ and $a_{u}$ in Proposition 1, we get:

1. Fix $\tau_{e} \in(0, \infty)$, as $\tau_{u} \rightarrow \infty$, then $a_{v}=\frac{\tau_{e}+\tau_{\varepsilon}}{\tau_{e}+\tau_{v}+\tau_{\varepsilon}}$ and $a_{u}=0$. Hence, $\alpha=0$.

2. Fix $\tau_{u} \in(0, \infty)$, as $\tau_{e} \rightarrow \infty$, then $a_{v}=\frac{\tau_{u}+\tau_{\varepsilon}\left(\alpha \sqrt{\tau_{u}}+1\right)^{2}}{\tau_{u}+\left(\alpha \sqrt{\tau_{u}}+1\right)^{2}\left(\tau_{v}+\tau_{\varepsilon}\right)}$ and $a_{u}=\frac{\sqrt{\tau_{u}}\left(\alpha \sqrt{\tau_{u}}+1\right)}{\tau_{u}+\left(\alpha \sqrt{\tau_{u}}+1\right)^{2}\left(\tau_{v}+\tau_{\varepsilon}\right)}$. Inserting the expressions of $a$ 's into $\alpha \equiv \frac{a_{u}}{a_{v}}$ and simplifying yields the following cubic equation that determines the value of $\alpha: \tau_{u} \tau_{\varepsilon} \alpha^{3}+2 \sqrt{\tau_{u}} \tau_{\varepsilon} \alpha^{2}+\tau_{\varepsilon} \alpha-\sqrt{\tau_{u}}=0$.

3. As both $\tau_{u} \rightarrow \infty$ and $\tau_{e} \rightarrow \infty$, then $a_{v}=1$ and $a_{u}=0$. Hence, the price function converges almost surely to $\tilde{p}^{R E E}=\tilde{v}$ and $\tilde{s}_{p, i}=\tilde{s}_{p}$. QED. 


\section{Proof of Proposition 2}

Return predictiveness $m$ is given by

$$
\begin{aligned}
m & =\frac{\operatorname{Cov}(\tilde{v}-\tilde{p}, \tilde{p})}{\operatorname{Var}(\tilde{p})}=\frac{\frac{a_{v}\left(1-a_{v}\right)}{\tau_{v}}-\frac{a_{u}^{2}}{\tau_{u}}}{\frac{a_{v}^{2}}{\tau_{v}}+\frac{a_{u}^{2}}{\tau_{u}}} \\
& =\frac{\tau_{v}\left(\tau_{\varepsilon} \tau_{e}^{2}\left(\alpha \sqrt{\tau_{u}}+1\right)^{4}+2 \tau_{e} \tau_{u} \tau_{\varepsilon}\left(\alpha \sqrt{\tau_{u}}+1\right)^{2}+\tau_{u}^{2}\left(\tau_{\varepsilon}+\tau_{e}\right)\right)}{\left(\tau_{e} \tau_{u}+\tau_{\varepsilon}\left(\tau_{u}+\tau_{e}\left(\alpha \sqrt{\tau_{u}}+1\right)^{2}\right)\right)^{2}+\tau_{e}^{2} \tau_{u} \tau_{v}\left(\alpha \sqrt{\tau_{u}}+1\right)^{2}}>0
\end{aligned}
$$

where $a_{v}$ and $a_{u}$ are the price coefficients in Proposition 1. QED.

\section{Proof of Proposition 3}

(a) Price informativeness

(1) Using equation (13), we can derive

$$
\frac{d \alpha}{d \tau_{u}}=\frac{-\left(\tau_{e} \tau_{\varepsilon} \alpha^{3}+2 \tau_{e}\left(\sqrt{\tau_{u}}\right)^{-1} \tau_{\varepsilon} \alpha^{2}+\tau_{\varepsilon} \alpha-\tau_{e}\left(\sqrt{\tau_{u}}\right)^{-1}\right)}{3 \tau_{e} \tau_{u} \tau_{\varepsilon} \alpha^{2}+4 \tau_{e} \sqrt{\tau_{u}} \tau_{\varepsilon} \alpha+\tau_{e} \tau_{\varepsilon}+\tau_{u} \tau_{\varepsilon}} .
$$

The sign of equation (26) is determined by its numerator, and we can conclude that $\lim _{\tau_{u} \rightarrow 0} \frac{d \alpha}{d \tau_{u}}>0$.

(b) Return volatility

(1) Return volatility is given by

$$
\sigma(\tilde{v}-\tilde{p})=\sqrt{\operatorname{Var}(\tilde{v}-\tilde{p})}
$$

where

$$
\operatorname{Var}(\tilde{v}-\tilde{p})=\frac{\left(1-a_{v}\right)^{2}}{\tau_{v}}+\frac{a_{u}^{2}}{\tau_{u}} .
$$

Using equation $(27)$, we can calculate the $\lim _{\tau_{u} \rightarrow \infty} \operatorname{Var}(\tilde{v}-\tilde{p})=\frac{\tau_{v}}{\left(\tau_{e}+\tau_{v}+\tau_{\varepsilon}\right)^{2}}$.

(2) Using equation (27), we can calculate the $\lim _{\tau_{u} \rightarrow 0} \operatorname{Var}(\tilde{v}-\tilde{p})=\frac{\tau_{e}^{2} \tau_{v}}{\left(\tau_{e} \tau_{v}+\tau_{e} \tau_{\varepsilon}\right)^{2}}$.

(c) Trading Volume

(1) Trading volume is given by

$$
\text { Volume }=\frac{1}{\gamma} \sqrt{\frac{2}{\pi} \operatorname{Var}\left[\gamma D\left(\tilde{p} ; \tilde{s}_{i}, \tilde{s}_{p, i}\right)\right]}
$$


where $\sigma_{y}^{2}=\operatorname{Var}\left[\gamma D\left(\tilde{p} ; \tilde{s}_{i}, \tilde{s}_{p, i}\right)\right]$ is given by (29). Taking the limit of this expression, we get

$$
\lim _{\tau_{u \rightarrow \infty}} \text { Volume }=\frac{1}{\gamma} \sqrt{\frac{2}{\pi}\left(\tau_{e}+\tau_{\varepsilon}\right)} .
$$

(2) Taking the limit of (28), we get

$$
\lim _{\tau_{u} \rightarrow 0} \text { Volume }=\frac{1}{\gamma} \sqrt{\frac{2}{\pi} \tau_{\varepsilon}}
$$

\section{E Proof of Proposition 4}

(a) Price informativeness

(1) Using equation (13), we can derive

$$
\begin{aligned}
\frac{d \alpha}{d \tau_{e}} & =\frac{-\left(\tau_{u} \tau_{\varepsilon} \alpha^{3}+2 \sqrt{\tau_{u}} \tau_{\varepsilon} \alpha^{2}+\tau_{\varepsilon} \alpha-\sqrt{\tau_{u}}\right)}{3 \tau_{e} \tau_{u} \tau_{\varepsilon} \alpha^{2}+4 \tau_{e} \sqrt{\tau_{u}} \tau_{\varepsilon} \alpha+\tau_{e} \tau_{\varepsilon}+\tau_{u} \tau_{\varepsilon}} \\
= & \frac{-\frac{\tau_{u} \tau_{\varepsilon} \alpha}{\tau_{e}}}{3 \tau_{e} \tau_{u} \tau_{\varepsilon} \alpha^{2}+4 \tau_{e} \sqrt{\tau_{u}} \tau_{\varepsilon} \alpha+\tau_{e} \tau_{\varepsilon}+\tau_{u} \tau_{\varepsilon}}<0
\end{aligned}
$$

where the second equality arises from using (13).

(b) Return volatility

(1) Using equation (27), we can calculate

$$
\lim _{\tau_{e} \rightarrow \infty} \operatorname{Var}(\tilde{v}-\tilde{p})=\frac{\left(\alpha \sqrt{\tau_{u}}+1\right)^{2}\left(\tau_{v} \alpha^{2} \tau_{u}+2 \tau_{v} \alpha \sqrt{\tau_{u}}+\tau_{u}+\tau_{v}\right)}{\left(\tau_{v}+\tau_{\varepsilon}+\tau_{u}+\alpha^{2} \tau_{u} \tau_{v}+\alpha^{2} \tau_{u} \tau_{\varepsilon}+2 \alpha \sqrt{\tau_{u}} \tau_{v}+2 \alpha \sqrt{\tau_{u}} \tau_{\varepsilon}\right)^{2}} .
$$

(2) Using equation (27), we can calculate the $\lim _{\tau_{e} \rightarrow 0} \operatorname{Var}(\tilde{v}-\tilde{p})=\frac{\tau_{u}^{2} \tau_{v}}{\left(\tau_{u} \tau_{v}+\tau_{u} \tau_{\varepsilon}\right)^{2}}$.

(c) Trading Volume

(1) Taking the limit of (28), we get

$$
\lim _{\tau_{e} \rightarrow \infty} \text { Volume }=\frac{1}{\gamma} \sqrt{\frac{2}{\pi} \tau_{\varepsilon}}
$$

(2) Taking the limit of (28), we get

$$
\lim _{\tau_{e} \rightarrow 0} \text { Volume }=\frac{1}{\gamma} \sqrt{\frac{2}{\pi} \tau_{\varepsilon}}
$$




\section{F Certainty Equivalent $W\left(\tau_{u, i}, \tau_{e, i} ; \tau_{u}, \tau_{e}\right)$ in Section $\mathbf{5 . 2}$}

At date- 0 , trader $i$ chooses $\tau_{u, i}$ and $\tau_{e, i}$ to maximize $E\left[-e^{-\gamma D\left(\tilde{p} ; \tilde{s}_{i}, \tilde{s}_{p, i}\right)(\tilde{v}-\tilde{p})+\gamma C\left(\tau_{u, i}, \tau_{e, i}\right)}\right]$. We will use the formula:

$$
\mathbf{E}\left[e^{-x y}\right]=\frac{1}{\sqrt{\left(1+\sigma_{x y}\right)^{2}-\sigma_{x}^{2} \sigma_{y}^{2}}},
$$

where $x \sim N\left(0, \sigma_{x}^{2}\right), y \sim N\left(0, \sigma_{y}^{2}\right)$ and $\operatorname{Cov}(x, y)=\sigma_{x y}$.

Define

$$
x=\tilde{v}-\tilde{p}, y=\gamma D\left(\tilde{p} ; \tilde{s}_{i}, \tilde{s}_{p, i}\right) .
$$

The certainty equivalent is:

$$
\begin{aligned}
W\left(\tau_{u, i}, \tau_{e, i} ; \tau_{u}, \tau_{e}\right) & =-\frac{1}{\gamma} \ln \left(-U_{i}\right) \\
& =\frac{1}{2 \gamma} \ln \left[\left(1+\sigma_{v-p, \gamma D_{i}}\right)^{2}-\sigma_{v-p}^{2} \sigma_{\gamma D_{i}}^{2}\right]-C\left(\tau_{u, i}, \tau_{e, i}\right) .
\end{aligned}
$$

Very intuitive: The first covariance term $\left(1+\sigma_{v-p, \gamma D_{i}}\right)^{2}$ is the benefit of beating the market, and the second term $\sigma_{v-p}^{2} \sigma_{\gamma D_{i}}^{2}$ of variance products is the cost of excess trading. The relevant terms are given by:

$$
\begin{aligned}
\sigma_{x}^{2} & =\frac{\left(1-a_{v}\right)^{2}}{\tau_{v}}+a_{u}^{2}, \\
\sigma_{y}^{2} & =\frac{\left(\frac{\left(\beta_{s, i}+\beta_{p, i}-a_{v}\right)^{2}}{\tau_{v}}+\left(\beta_{p, i} \alpha+\frac{\beta_{p, i}}{\sqrt{\tau_{u, i}}}-a_{u}\right)^{2}+\frac{\beta_{s, i}^{2}}{\tau_{\varepsilon}}+\frac{\beta_{p, i}^{2}}{\tau_{e, i}}\right)}{V\left(v \mid s_{i}, s_{p . i}\right)^{2}}, \\
\sigma_{x y} & =\frac{\frac{\left(1-a_{v}\right)\left(\beta_{s, i}+\beta_{p, i}-a_{v}\right)}{\tau_{v}}-a_{u}\left(\beta_{p, i} \alpha+\frac{\beta_{p, i}}{\sqrt{\tau_{u, i}}}-a_{u}\right)}{V\left(v \mid s_{i}, s_{p . i}\right)},
\end{aligned}
$$

where $a_{v}$ and $a_{u}$ are the price coefficients in Proposition 1 and $\beta_{s, i}$ and $\beta_{p, i}$ are the coefficients of the conditional expectation

$$
E\left(\tilde{v} \mid \tilde{s}_{i}, \tilde{s}_{p, i}\right)=\beta_{s, i} \tilde{s}_{i}+\beta_{p, i} \tilde{s}_{p, i}
$$

which are given by equation (11).

\section{G Proof of Proposition 5}

Let us define

$$
\Phi\left(\tau_{u}\right) \equiv \phi\left(\tau_{u}\right)+C^{\prime}\left(\tau_{u}\right)
$$


The limits of the function $\phi\left(\tau_{u}\right)$ are given by:

$$
\begin{aligned}
\lim _{\tau_{u} \rightarrow 0} \phi\left(\tau_{u}\right) & =\frac{\tau_{v}-\tau_{\varepsilon}}{\gamma\left(\tau_{v}^{2}+\tau_{v} \tau_{\varepsilon}+\tau_{\varepsilon}^{2}\right)}, \\
\lim _{\tau_{u} \rightarrow \infty} \phi\left(\tau_{u}\right) & =0
\end{aligned}
$$

and the limits of its total derivative $\frac{d \phi\left(\tau_{u}\right)}{d \tau_{u}}$ are given by

$$
\begin{aligned}
& \lim _{\tau_{u} \rightarrow 0} \frac{d \phi\left(\tau_{u}\right)}{d \tau_{u}}=\infty \\
& \lim _{\tau_{u} \rightarrow \infty} \frac{d \phi\left(\tau_{u}\right)}{d \tau_{u}}=0 .
\end{aligned}
$$

If $\tau_{v}>\tau_{\varepsilon}$, then $\lim _{\tau_{u} \rightarrow 0} \phi\left(\tau_{u}\right)>\lim _{\tau_{u} \rightarrow \infty} \phi\left(\tau_{u}\right)$. In addition, if $C^{\prime \prime}(0)$ is sufficiently small, then $\phi^{\prime}(0)=\Phi^{\prime}(0)-C^{\prime \prime}(0)>0$, and thus $\phi^{\prime}\left(\tau_{u}\right)>0$ for sufficiently small values of $\tau_{u}$.

\section{H Proof of Proposition 6}

Taking the first order condition with respect to $\tau_{e, i}$ from the certainty equivalent derived above in section $\mathrm{F}$ when $\tau_{u} \rightarrow \infty$ and setting $\tau_{e, i}=\tau_{e}$, we get:

$$
\frac{\partial W\left(\tau_{e} ; \tau_{e}\right)}{\partial \tau_{e, i}}=-\frac{2 \gamma \kappa_{e} \tau_{v}^{2}+2 \gamma \kappa_{e} \tau_{v} \tau_{\varepsilon}+2 \gamma \kappa_{e} \tau_{v} \tau_{e}-\tau_{v}+2 \gamma \kappa_{e} \tau_{\varepsilon}^{2}+4 \gamma \kappa_{e} \tau_{\varepsilon} \tau_{e}+2 \gamma \kappa_{e} \tau_{e}^{2}}{2 \gamma\left(\tau_{v}^{2}+\tau_{v} \tau_{\varepsilon}+\tau_{v} \tau_{e}+\tau_{\varepsilon}^{2}+2 \tau_{\varepsilon} \tau_{e}+\tau_{e}^{2}\right)}=0
$$

Since $\frac{\partial^{2} W\left(\tau_{e, i} ; \tau_{e}\right)}{\partial \tau_{e, i}^{2}}<0$, for small enough $\kappa_{e}$, there is a unique symmetric equilibrium with $\tau_{e}>0$ given by

$$
\tau_{e, i}^{*}=\tau_{e}^{*}=-\frac{\gamma^{(1 / 2)} \kappa_{e}^{(1 / 2)} \tau_{v}-\left(-\tau_{v}\left(3 \gamma \kappa_{e} \tau_{v}-2\right)\right)^{(1 / 2)}+2 \gamma^{(1 / 2)} \kappa_{e}^{(1 / 2)} \tau_{\varepsilon}}{2 \gamma^{(1 / 2)} \kappa_{e}^{(1 / 2)}} .
$$

Otherwise, there is a unique symmetric equilibrium with $\tau_{e, i}^{*}=\tau_{e}^{*}=0$.

\section{Proof of Proposition 7}

Using Bayes' rule, we can compute investors' beliefs given by equations (20) and (21). In a symmetric equilibrium with $\tau_{u, i}=\tau_{u}$ and $\tau_{e, i}=\tau_{e}$, all investors have the same conditional variance $\operatorname{Var}\left(\tilde{v} \mid \tilde{s}_{i}, \tilde{s}_{p, i}\right)$. Thus, inserting these two expressions into the demand (5), we can compute the expression of $D\left(\tilde{p} ; \tilde{s}_{i}, \tilde{s}_{p, i}\right)$, which is in turn inserted into (18) to compute the equilibrium price as a function of $\tilde{v}, \tilde{u}$ and $\tilde{q}$. Comparing coefficients with the conjectured price function (19), we can form a system of equations to determine the three unknown price coefficients 
$a_{v}, a_{u}$ and $a_{q}$ in Proposition 7. Inserting the expressions of $a$ 's into $\alpha \equiv \frac{a_{u}}{a_{v}}$ and simplifying yields the cubic (22) that determines the value of $\alpha$. The discriminant of the cubic (22) is negative. Thus, there exists a unique real root, which establishes the uniqueness of a financial market equilibrium.

Return predictiveness $m$ is given by

$$
m=\frac{\operatorname{Cov}(\tilde{v}-\tilde{p}, \tilde{p})}{\operatorname{Var}(\tilde{p})}=\frac{\operatorname{Cov}(\tilde{v}-\tilde{p}, \tilde{p})}{\operatorname{Var}(\tilde{p})}=\frac{\frac{a_{v}\left(1-a_{v}\right)}{\tau_{v}}-\frac{a_{u}^{2}}{\tau_{u}}-\frac{a_{q}^{2}}{\tau_{q}}}{\frac{a_{v}^{2}}{\tau_{v}}+\frac{a_{u}^{2}}{\tau_{u}}+\frac{a_{q}^{2}}{\tau_{q}}}
$$

The sign of $m$ is determined by a cubic equation $Q\left(\tau_{q}\right)=q_{3} \tau_{q}^{3}+q_{2} \tau_{q}^{2}+q_{1} \tau_{q}+q_{0}$. Since the discriminant of this cubic equation is positive, there are three real solutions to $Q\left(\tau_{q}\right)=0$. Also, the $\lim _{\tau_{q} \rightarrow \infty} m>0$. Hence, denoting $\hat{\tau}_{q}$ as the maximum real root to $Q\left(\tau_{q}\right)=0$, we can conclude that $m>0$ for any $\tau_{q}>\hat{\tau}_{q}$. QED.

\section{J Proof of Proposition 8}

See proof of Proposition 1 in Ganguli and Yang (2009) for price coefficients $a_{v}$ and $a_{q}$ and the properties of information acquisition. For the SUB equilibrium:

$$
\begin{aligned}
m & =\frac{\operatorname{Cov}(\tilde{v}-\tilde{p}, \tilde{p})}{\operatorname{Var}(\tilde{p})}=\frac{\frac{a_{v}\left(1-a_{v}\right)}{\tau_{v}}-\frac{a_{q}^{2}}{\tau_{q}}}{\frac{a_{v}^{2}}{\tau_{v}}+\frac{a_{q}^{2}}{\tau_{q}}} \\
& =-\frac{\gamma \tau_{v}\left(2 \gamma \tau_{\eta}+\gamma \tau_{q}-\tau_{q}\left(\gamma^{2}-4 \tau_{\eta} \tau_{\varepsilon}\right)^{1 / 2}\right)}{\left(2 \tau_{\eta}\right)\left(\beta^{4} \tau_{q}^{3}+2 \beta^{4} \tau_{q}^{2} \tau_{\eta}+\beta^{4} \tau_{q} \tau_{\eta}^{2}+2 \beta^{2} \tau_{q}^{2} \tau_{\varepsilon}+\tau_{v} \beta^{2} \tau_{q}^{2}+2 \beta^{2} \tau_{q} \tau_{\eta} \tau_{\varepsilon}+2 \tau_{v} \beta \gamma \tau_{q}+\tau_{v} \gamma^{2}+\tau_{q} \tau_{\varepsilon}^{2}\right)}<0
\end{aligned}
$$

For the COM equilibrium:

$$
\begin{aligned}
m & =\frac{\operatorname{Cov}(\tilde{v}-\tilde{p}, \tilde{p})}{\operatorname{Var}(\tilde{p})}=\frac{\frac{a_{v}\left(1-a_{v}\right)}{\tau_{v}}-\frac{a_{q}^{2}}{\tau_{q}}}{\frac{a_{v}^{2}}{\tau_{v}}+\frac{a_{q}^{2}}{\tau_{q}}} \\
& =-\frac{\gamma \tau_{v}\left(2 \gamma \tau_{\eta}+\gamma \tau_{q}+\tau_{q}\left(\gamma^{2}-4 \tau_{\eta} \tau_{\varepsilon}\right)^{1 / 2}\right)}{\left(2 \tau_{\eta}\right)\left(\beta^{4} \tau_{q}^{3}+2 \beta^{4} \tau_{q}^{2} \tau_{\eta}+\beta^{4} \tau_{q} \tau_{\eta}^{2}+2 \beta^{2} \tau_{q}^{2} \tau_{\varepsilon}+\tau_{v} \beta^{2} \tau_{q}^{2}+2 \beta^{2} \tau_{q} \tau_{\eta} \tau_{\varepsilon}+2 \tau_{v} \beta \gamma \tau_{q}+\tau_{v} \gamma^{2}+\tau_{q} \tau_{\varepsilon}^{2}\right)}<0
\end{aligned}
$$

\section{K Proof of Proposition 9}

Using Bayes' rule, we can compute investors' beliefs. Inserting the expressions for $E\left(\tilde{v} \mid \tilde{s}_{i}, \tilde{s}_{p, i}\right)$ and $\operatorname{Var}\left(\tilde{v} \mid \tilde{s}_{i}, \tilde{s}_{p, i}\right)$ into the demand $D\left(\tilde{p} ; \tilde{s}_{i}, \tilde{s}_{p}\right)$, which has the same expression as equation $(5)$. This demand is in turn inserted into the market clearing condition to compute the equilibrium price as a function of $\tilde{v}$ and $\tilde{q}$. Comparing coefficients with the conjectured price function (23), 
we can form a system of equations to determine the two unknown price coefficients $a_{v}$ and $a_{q}$ in Proposition 9. Inserting the expressions of $a$ 's into $\alpha \equiv \frac{a_{q}}{a_{v}}$ and simplifying yields a cubic equation of $\alpha$. The discriminant of the cubic is negative. Thus, there exists a unique real root given by $\alpha=-\gamma / \tau_{\varepsilon}$, which establishes the uniqueness of a financial market equilibrium. The true (econometrician's) distribution of prices is given by $\tilde{p}=a_{v} \tilde{v}+a_{q} \tilde{q}$, not $\tilde{p}=a_{v} \rho \tilde{v}+a_{v} \sqrt{\left(1-\rho^{2}\right)} \tilde{\phi}+$ $a_{q} \tilde{q}$. The latter is only under the beliefs of the investors, but not under the true distribution. Hence, return predictiveness $m$ is given by

$$
\begin{aligned}
m & =\frac{\operatorname{Cov}(\tilde{v}-\tilde{p}, \tilde{p})}{\operatorname{Var}(\tilde{p})}=\frac{\frac{\left(1-a_{v}\right) a_{v}}{\tau_{v}}-\frac{a_{q}^{2}}{\tau_{q}}}{\frac{\left(a_{v}\right)^{2}}{\tau_{v}}+\frac{a_{q}^{2}}{\tau_{q}}} \\
& =-\frac{\tau_{v}\left(\tau_{v} \gamma^{4}+\left(1-\rho^{2}\right) \gamma^{2} \tau_{q} \tau_{\varepsilon}^{2}-(1-\rho) \tau_{v} \gamma^{2} \tau_{q} \tau_{\varepsilon}-(1-\rho) \tau_{q}^{2} \tau_{\varepsilon}^{3}\right)}{\left(\tau_{q} \tau_{\varepsilon}^{2}+\tau_{v} \gamma^{2}\right)\left(\tau_{v} \gamma^{2}+\left(1-\rho^{2}\right) \tau_{q} \tau_{\varepsilon}^{2}+\tau_{q} \tau_{v} \rho \tau_{\varepsilon}\right)}
\end{aligned}
$$

The limits of $m$ are given by

$$
\lim _{\gamma \rightarrow 0} m>0 \text { and } \lim _{\gamma \rightarrow \infty} m<0
$$

Hence, if $\gamma$ is sufficiently small, then $m>0$, but if if $\gamma$ is sufficiently large, then $m<0$. Trading volume in this model is $\int_{0}^{1}\left|D\left(p ; s_{i}, s_{p, i}\right)\right| d i$ and is given by

$$
\text { Volume }=\frac{1}{\gamma} \sqrt{\frac{2}{\pi} \frac{\left(\gamma^{2} \tau_{v}+2 \tau_{q} \tau_{\varepsilon}^{2}-2 \rho \tau_{q} \tau_{\varepsilon}^{2}+\tau_{q} \tau_{v} \tau_{\varepsilon}\right)}{\tau_{q} \tau_{v}}}
$$

From this equation, it immediately follows that volume is decreasing in $\rho$. QED

\section{Proof of Proposition 10}

See Proof of Proposition 2 in Eyster, Rabin and Vayanos (2019).

\section{Proof of Proposition 11}

Using Bayes' rule, we can compute investors' beliefs. Inserting the expressions for $E\left(\tilde{v} \mid \tilde{s}_{i}, \tilde{s}_{p, i}\right)$ and $\operatorname{Var}\left(\tilde{v} \mid \tilde{s}_{i}, \tilde{s}_{p, i}\right)$ into the demand (24), we can compute the expression of $D\left(\tilde{p} ; \tilde{s}_{i}, \tilde{s}_{p}\right)$, which is in turn inserted into the market clearing condition to compute the equilibrium price as a function of $\tilde{v}, \tilde{x}$ and $\tilde{q}$. Comparing coefficients with the conjectured price function (25), we can form a system of equations to determine the three unknown price coefficients $a_{v}, a_{x}$ and $a_{q}$ in Proposition 11. Inserting the expressions of $a$ 's into $\alpha \equiv \frac{a_{q}}{a_{v}}, \theta \equiv \frac{a_{x}}{a_{v}}$ and simplifying yields the cubic equation for $\alpha$ and $\theta=-\alpha$. 


\section{N Proof of Corollary 2}

We can obtain the unknown price coefficients by taking the limit of the price coefficients derived in Proposition 11. The asset price is isomorphic to the standard REE equilibrium and therefore, there is no momentum $m<0$. The expression for momentum is the same as in the proof of Proposition 9 with $\rho=1$. QED. 